\title{
Intramolecular Heck Reactions of Unactivated Alkyl Halides
}

\author{
Luke Firmansjah and Gregory C. Fu* \\ Department of Chemistry, Massachusetts Institute of Technology, \\ Cambridge, Massachusetts 02139
}

\section{Supporting Information}

\section{General}

$\mathrm{Pd}_{2}(\mathrm{MeO}-\mathrm{dba})_{3}$ was prepared as described in Section II. SIMes $\bullet \mathrm{HBF}_{4}(\mathrm{Strem}), \mathrm{KO} t$ $\mathrm{Bu}$ (Strem), $\mathrm{Cs}_{2} \mathrm{CO}_{3}$ (Strem), $\mathrm{K}_{3} \mathrm{PO}_{4}$ (Strem), anhydrous $\mathrm{CH}_{3} \mathrm{CN}$ (Fluka), anhydrous $\mathrm{NMP}$ (Fluka), and $\mathrm{AgNO}_{3}$ on silica gel (10 wt\%; Aldrich) were used as received.

\section{Preparation of $\mathbf{P d}_{2}(\mathrm{MeO}-\mathrm{dba})_{3}$}

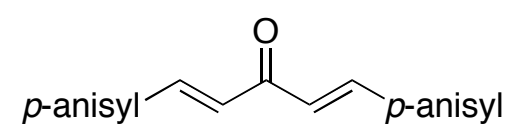

(1E,4E)-1,5-Bis(4-methoxyphenyl)penta-1,4-dien-3-one ("MeO-dba”) [37951-12-5]. Prepared according to the procedure of Fairlamb. ${ }^{1}$ The material was recrystallized from 1:3 $\mathrm{CH}_{2} \mathrm{Cl}_{2}: \mathrm{Et}_{2} \mathrm{O}$ ). Yield: $70 \%$. $\mathrm{mp} 122-123{ }^{\circ} \mathrm{C}$.

$\mathbf{P d}_{\mathbf{2}}(\mathrm{MeO}-\mathrm{dba})_{3}$ [130610-16-1]. This procedure was adapted from a report of Beller. ${ }^{2}$ Anhydrous $\mathrm{NaOAc}(1.95 \mathrm{~g}, 23.8 \mathrm{mmol}, 8.0$ equiv) and $\mathrm{MeO}-\mathrm{dba}(2.88 \mathrm{~g}, 9.8 \mathrm{mmol}, 3.3$ equiv) were added to a $250-\mathrm{mL}$ two-neck round-bottom flask, which was purged with argon for $10 \mathrm{~min}$. Dry $\mathrm{MeOH}(70 \mathrm{~mL})$ was added, and the mixture was stirred for 10 min at $50{ }^{\circ} \mathrm{C} . \mathrm{PdCl}_{2}(0.53 \mathrm{~g}, 3.0 \mathrm{mmol}, 1.0$ equiv $)$ was then added under a positive flow of argon, and the mixture was stirred at $40^{\circ} \mathrm{C}$ for $4 \mathrm{~h}$. The mixture was then cooled to 0 ${ }^{\circ} \mathrm{C}$ in an ice bath and filtered. The solid was washed with $\mathrm{H}_{2} \mathrm{O}(2 \times 15 \mathrm{~mL})$ and acetone $(2 \times 15 \mathrm{~mL})$, and the resulting dark-purple solid was dried in vacuo overnight. Yield: $2.1 \mathrm{~g}(65 \%)$.

mp 144-146 ${ }^{\circ} \mathrm{C}$ (lit: $\left.141-143{ }^{\circ} \mathrm{C}\right)$;

Anal. Calcd. for $\mathrm{C}_{57} \mathrm{H}_{54} \mathrm{O}_{9} \mathrm{Pd}_{2}$ (1095.87), C 62.47, H 4.97, Pd 19.42; Found, C 65.46, H 5.15, Pd 13.90. 


\section{Intramolecular Alkyl-Heck Reactions}

General procedure for alkyl bromides (Table 2). An oven-dried 25-mL Schlenk flask was purged with argon for $10 \mathrm{~min}$, then charged with $\mathrm{Pd}_{2}(\mathrm{MeO}-\mathrm{dba})_{3}(27.4 \mathrm{mg}$, $0.025 \mathrm{mmol}, 0.050$ equiv), SIMes $\cdot \mathrm{HBF}_{4}(39.4 \mathrm{mg}, 0.10 \mathrm{mmol}, 0.20$ equiv), KOt-Bu (11.2 $\mathrm{mg}, 0.10 \mathrm{mmol}, 0.20$ equiv), and $\mathrm{Cs}_{2} \mathrm{CO}_{3}(179 \mathrm{mg}, 0.55 \mathrm{mmol}, 1.1$ equiv) (for Entry 9: $\mathrm{K}_{3} \mathrm{PO}_{4}(117 \mathrm{mg})$ ). The flask was then purged with argon for an additional $5 \mathrm{~min}$, with stirring. Dry $\mathrm{CH}_{3} \mathrm{CN}(5 \mathrm{~mL})$ was added, and the mixture was stirred under argon for 10 min, at which time it was dark orange-brown. The electrophile was then added via a gastight syringe $(0.50 \mathrm{mmol}, 1.0$ equiv), and the Schlenk flask was sealed. The reaction mixture was stirred for $16 \mathrm{~h}$ at $65{ }^{\circ} \mathrm{C}$.

For entries 2-8, the mixture was allowed to cool to room temperature, and then it was passed through a pad of silica gel, washing with $\mathrm{Et}_{2} \mathrm{O}$. Removal of the solvent on a rotary evaporator provided an orange-brown residue, which was purified by column chromatography ( $10 \% \mathrm{w} / \mathrm{w} \mathrm{AgNO}_{3}$ on $\mathrm{SiO}_{2} ; 10 \mathrm{~g} / \mathrm{mmol}$ product).

Because the reaction products for entries 1 and 9 are slightly volatile, removal of the $\mathrm{CH}_{3} \mathrm{CN}$ on a rotary evaporator led to a lower yield. Therefore, the cooled reaction mixture was diluted with $\mathrm{H}_{2} \mathrm{O}(100 \mathrm{~mL})$ and pentane $(50 \mathrm{~mL})$. The organic layer was separated from the aqueous layer and passed through a plug of silica gel, washing with pentane $(20 \mathrm{~mL})$. The pentane was removed by warming at atmospheric pressure (distillation), and the residue was purified by chromatography $\left(10 \% \mathrm{w} / \mathrm{w} \mathrm{AgNO}_{3}\right.$ on $\mathrm{SiO}_{2} ; 10 \mathrm{~g} / \mathrm{mmol}$ product).

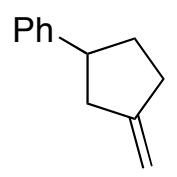

(3-Methylenecyclopentyl)benzene (Table 2, entry 1) [122833-99-2].

Chromatography (100\% pentane) furnished the desired product as a colorless oil. Run 1: $56.7 \mathrm{mg}$ (72\%); Run 2: $58.4 \mathrm{mg}(74 \%)$.

${ }^{1} \mathrm{H} \mathrm{NMR}\left(\mathrm{CDCl}_{3}, 300 \mathrm{MHz}\right)$ 8 7.35-7.19 (m, 5H), 4.95-4.85 (m, 2H), 3.21-3.08 (m, $\left.1 \mathrm{H}\right)$, $2.75(\mathrm{ddt}, J=16.5 \mathrm{~Hz}, 7.1 \mathrm{~Hz}, 1.6 \mathrm{~Hz}, 1 \mathrm{H}), 2.60-2.45(\mathrm{~m}, 1 \mathrm{H}), 2.43-2.34(\mathrm{~m}, 2 \mathrm{H}), 2.21-2.10$ (m, 1H), 1.82-1.67 (m, 1H);

${ }^{13} \mathrm{C} \mathrm{NMR}\left(\mathrm{CDCl}_{3}, 75 \mathrm{MHz}\right) \delta 152.4,145.2,128.6,127.2,126.3,105.6,45.8,41.4,34.7$, 32.8 ;

IR (film) 3066, 3029, 2948, 2250, 1657, 1603, 1541, 1494, 1451, 1434, 1265, 1031, 909, $878,734,699,650 \mathrm{~cm}^{-1}$;

GCMS (EI) calcd for $\mathrm{C}_{12} \mathrm{H}_{14} 158[\mathrm{M}]^{+}$, found 158 . 


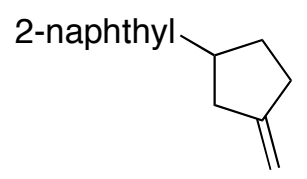

2-(3-Methylenecyclopentyl)naphthalene (Table 2, entry 2). Chromatography (100\% hexanes) furnished the desired product as a colorless oil. Run 1: $83.2 \mathrm{mg}(80 \%)$; Run 2: $79.0 \mathrm{mg}(76 \%)$.

${ }^{1} \mathrm{H}$ NMR $\left(\mathrm{CDCl}_{3}, 500 \mathrm{MHz}\right) \delta$ 7.83-7.76 (m, 3H), $7.67(\mathrm{~s}, 1 \mathrm{H}), 7.49-7.39(\mathrm{~m}, 3 \mathrm{H}), 5.00-$ $4.96(\mathrm{~m}, 1 \mathrm{H}), 4.96-4.93(\mathrm{~m}, 1 \mathrm{H}), 3.34(\mathrm{tt}, J=10.3 \mathrm{~Hz}, 7.2 \mathrm{~Hz}, 1 \mathrm{H}), 2.84(\mathrm{dd}, J=16.0 \mathrm{~Hz}, 7.3$ $\mathrm{Hz}, 1 \mathrm{H}), 2.63-2.55(\mathrm{~m}, 1 \mathrm{H}), 2.56-2.49(\mathrm{~m}, 1 \mathrm{H}), 2.51-2.42(\mathrm{~m}, 1 \mathrm{H}), 2.28-2.21(\mathrm{~m}, 1 \mathrm{H}), 1.88$ (dddd, $J=12.1 \mathrm{~Hz}, 10.3 \mathrm{~Hz}, 10.3 \mathrm{~Hz}, 8.3 \mathrm{~Hz}, 1 \mathrm{H}$ );

${ }^{13} \mathrm{C} \mathrm{NMR}\left(\mathrm{CDCl}_{3}, 75 \mathrm{MHz}\right) \delta 152.8,143.2,134.2,132.9,128.6,128.3,126.7,126.6,125.9$, 125.6, 106.2, 46.4, 41.8, 35.2, 33.4;

IR (film) 3054, 2919, 2850, 1657, 1633, 1601, 1543, 1508, 1433, 1373, 1269, 1019, 876, $853,816,744 \mathrm{~cm}^{-1}$;

GCMS (EI) calcd for $\mathrm{C}_{16} \mathrm{H}_{16} 208[\mathrm{M}]^{+}$, found 208 .

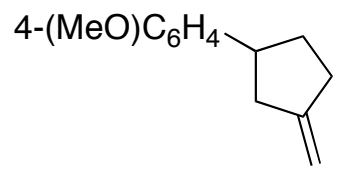

\section{1-Methoxy-4-(3-methylenecyclopentyl)benzene (Table 2, entry 3).}

Chromatography (50:1 hexanes: $\mathrm{Et}_{2} \mathrm{O}$ ) furnished the desired product as a colorless oil. Run 1: $76.1 \mathrm{mg}(81 \%)$; Run 2: $78.0 \mathrm{mg}(83 \%)$.

${ }^{1} \mathrm{H} \mathrm{NMR}\left(\mathrm{CDCl}_{3}, 300 \mathrm{MHz}\right)$ 8 7.21-7.15 (m, 2H), 6.88-6.83 (m, 2H), 4.95-4.91 (m, 1H), 4.92-4.88 (m, 1H), 3.80 (s, 3H), 3.10 (dddd, $J=10.6 \mathrm{~Hz}, 10.6 \mathrm{~Hz}, 6.6 \mathrm{~Hz}, 6.6 \mathrm{~Hz}, 1 \mathrm{H}), 2.76-$ $2.70(\mathrm{~m}, 1 \mathrm{H}), 2.56-2.49(\mathrm{~m}, 1 \mathrm{H}), 2.44-2.34(\mathrm{~m}, 2 \mathrm{H}), 2.16-2.10(\mathrm{~m}, 1 \mathrm{H}), 1.72(\mathrm{dddd}, J=8.7$ $\mathrm{Hz}, 10.7 \mathrm{~Hz}, 10.7 \mathrm{~Hz}, 12.5 \mathrm{~Hz}, 1 \mathrm{H})$;

${ }^{13} \mathrm{C} \mathrm{NMR}\left(\mathrm{CDCl}_{3}, 75 \mathrm{MHz}\right) \delta 158.6,152.9,137.8,128.6,114.4,106.0,56.0,45.5,42.0$, 35.4, 33.2;

IR (film) 3070, 3033, 2998, 2950, 2907, 2835, 1655, 1612, 1583, 1514, 1464, 1441, 1361, $1304,1282,1249,1179,1108,1039,875,828,668 \mathrm{~cm}^{-1}$;

GCMS (EI) calcd for $\mathrm{C}_{13} \mathrm{H}_{16} \mathrm{O} 188[\mathrm{M}]^{+}$, found 188 . 


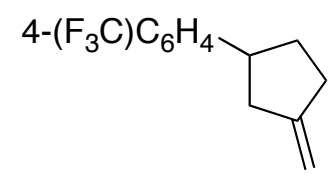

\section{1-(3-Methylenecyclopentyl)-4-(trifluoromethyl)benzene (Table 2, entry 4).}

Chromatography (100:1 hexanes:Et $\left.{ }_{2} \mathrm{O}\right)$ furnished the desired product as a colorless oil. Run 1: $88.1 \mathrm{mg}$ (78\%); Run 2: $90.4 \mathrm{mg}(80 \%)$.

${ }^{1} \mathrm{H}$ NMR $\left(\mathrm{CDCl}_{3}, 300 \mathrm{MHz}\right) \delta 7.55(\mathrm{~d}, J=8.2 \mathrm{~Hz}, 2 \mathrm{H}), 7.36(\mathrm{~d}, J=8.1 \mathrm{~Hz}, 2 \mathrm{H}), 4.96-$ $4.91(\mathrm{~m}, 2 \mathrm{H}), 3.15-3.12(\mathrm{~m}, 1 \mathrm{H}), 2.78(\mathrm{ddt}, J=17.0 \mathrm{~Hz}, 7.4 \mathrm{~Hz}, 1.5 \mathrm{~Hz}, 1 \mathrm{H}), 2.62-2.36(\mathrm{~m}$, $3 \mathrm{H}), 2.21-2.15(\mathrm{~m}, 1 \mathrm{H}), 1.76(\mathrm{~m}, 1 \mathrm{H})$;

${ }^{13} \mathrm{C} \mathrm{NMR}\left(\mathrm{CDCl}_{3}, 75 \mathrm{MHz}\right) \delta 151.5,149.4,127.6,125.5(\mathrm{q}, J=4.0 \mathrm{~Hz}), 106.1,45.6,41.2$, 34.6, 32.6;

${ }^{19} \mathrm{~F}$ NMR $\left(\mathrm{CDCl}_{3}, 282 \mathrm{MHz}\right) \delta-62.9$;

IR (film) 2917, 2850, 1659, 1620, 1579, 1543, 1462, 1421, 1327, 1164, 1124, 1069, 1018, $880,838 \mathrm{~cm}^{-1}$;

GCMS (EI) calcd for $\mathrm{C}_{13} \mathrm{H}_{13} \mathrm{~F}_{3} 226[\mathrm{M}]^{+}$, found 226 .

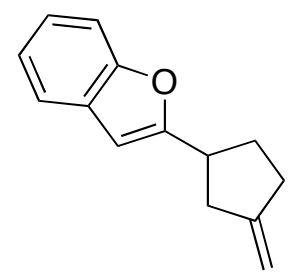

2-(3-Methylenecyclopentyl)benzofuran (Table 2, entry 5). Chromatography (100:1 hexanes: $\mathrm{Et}_{2} \mathrm{O}$ ) furnished the desired product as a colorless oil. Run 1: $82.2 \mathrm{mg}(83 \%)$; Run 2: $81.2 \mathrm{mg}(82 \%)$.

${ }^{1} \mathrm{H}$ NMR $\left(\mathrm{CDCl}_{3}, 300 \mathrm{MHz}\right) \delta$ 7.51-7.47 (m, 1H), 7.44-7.40 (m, 1H), $7.22(\mathrm{td}, J=7.2 \mathrm{~Hz}$, $1.8 \mathrm{~Hz}, 1 \mathrm{H}), 7.17(\mathrm{td}, J=7.2 \mathrm{~Hz} 1.4 \mathrm{~Hz}, 1 \mathrm{H}), 6.41(\mathrm{t}, J=1.0 \mathrm{~Hz}, 1 \mathrm{H}), 4.97-4.93(\mathrm{~m}, 1 \mathrm{H})$, 4.95-4.90 (m, 1H), 3.40-3.29 (m, 1H), $2.79(\mathrm{dd}, J=16.4 \mathrm{~Hz}, 7.7 \mathrm{~Hz}, 1 \mathrm{H}), 2.65-2.35(\mathrm{~m}, 3 \mathrm{H})$, 2.24-2.17 (m, 1H), $1.95(\mathrm{dq}, J=12.4 \mathrm{~Hz}, 8.9 \mathrm{~Hz}, 1 \mathrm{H})$;

${ }^{13} \mathrm{C} \mathrm{NMR}\left(\mathrm{CDCl}_{3}, 75 \mathrm{MHz}\right) \delta 162.5,155.3,151.6,129.5,123.9,123.1,121.0,111.5,106.7$, 101.5, 39.8, 39.1, 32.8, 32.5;

IR (film) 3071, 2918, 2850, 1659, 1600, 1586, 1455, 1434, 1254, 1179, 1104, 1011, 965 , 926, 909, 881, 797, 750, $739 \mathrm{~cm}^{-1}$;

GCMS (EI) calcd for $\mathrm{C}_{14} \mathrm{H}_{14} \mathrm{O} 198[\mathrm{M}]^{+}$, found 198 . 


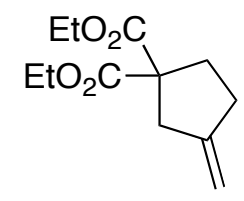

Diethyl 3-methylenecyclopentane-1,1-dicarboxylate (Table 2, entry 6) [137435-462]. Chromatography $\left(20: 1\right.$ hexanes: $\left.\mathrm{Et}_{2} \mathrm{O}\right)$ furnished the desired product as a colorless oil. Run 1: $96.1 \mathrm{mg}$ (85\%); Run 2: $97.2 \mathrm{mg}(86 \%)$.

${ }^{1} \mathrm{H}$ NMR $\left(\mathrm{CDCl}_{3}, 300 \mathrm{MHz}\right) \delta$ 4.95-4.82 (m, 2H), $4.18(\mathrm{q}, J=7.1 \mathrm{~Hz}, 4 \mathrm{H}), 2.92-2.87(\mathrm{~m}$, 2H), 2.46-2.37 (m, 2H), $2.25(\mathrm{td}, J=7.7 \mathrm{~Hz}, 0.9 \mathrm{~Hz}, 2 \mathrm{H}), 1.24(\mathrm{t}, J=7.1 \mathrm{~Hz}, 6 \mathrm{H})$;

${ }^{13} \mathrm{C} \mathrm{NMR}\left(\mathrm{CDCl}_{3}, 75 \mathrm{MHz}\right) \delta 172.0,148.7,107.1,61.7,60.4,40.9,39.9,31.5,14.3$;

IR (film) 3077, 2984, 2939, 2875, 1732, 1663, 1465, 1446, 1390, 1367, 1299, 1274, 1252, 1203, 1181, 1157, 1097, 1071, 1041, 1019, 934, 882, $862 \mathrm{~cm}^{-1}$;

GCMS (EI) calcd for $\mathrm{C}_{12} \mathrm{H}_{18} \mathrm{O}_{4} 226[\mathrm{M}]^{+}$, found 226 .

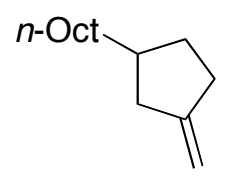

1-Methylene-3-octylcyclopentane (Table 2, entry 7). Chromatography (100\% hexanes) furnished the desired product as a colorless oil. Run 1: $73.7 \mathrm{mg}(76 \%)$; Run 2: $71.8 \mathrm{mg}(74 \%)$.

${ }^{1} \mathrm{H}$ NMR $\left(\mathrm{CDCl}_{3}, 300 \mathrm{MHz}\right) \delta 4.84-4.81(\mathrm{~m}, 1 \mathrm{H}), 4.82-4.79(\mathrm{~m}, 1 \mathrm{H}), 2.47-2.44(\mathrm{~m}, 1 \mathrm{H})$, 2.39-2.33 (m, 1H), 2.28-2.21 (m, 1H), 1.90-1.83 (m, 3H), 1.34-1.28 (m, 15H), $0.89(\mathrm{t}, J=7.0$ $\mathrm{Hz}, 3 \mathrm{H})$;

${ }^{13} \mathrm{C} \mathrm{NMR}\left(\mathrm{CDCl}_{3}, 75 \mathrm{MHz}\right) \delta 153.5,104.9,40.7,40.2,35.7,33.1,32.6,32.2,30.1,29.9$, 29.6, 28.7, 22.9, 14.4;

IR (film) 3071, 2923, 2854, 1755, 1658, 1466, 1433, 1378, 875, $722 \mathrm{~cm}^{-1}$;

GCMS (EI) calcd for $\mathrm{C}_{14} \mathrm{H}_{26} 194[\mathrm{M}]^{+}$, found 194.

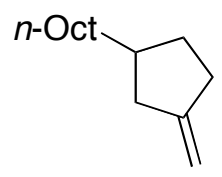

1-Methylene-3-octylcyclopentane (Table 2, entry 8). Chromatography (100\% hexanes) furnished the desired product as a colorless oil. Run 1: $71.8 \mathrm{mg}$ (74\%); Run 2: $69.8 \mathrm{mg}(72 \%)$.

For characterization data, see Table 2, entry 7. 


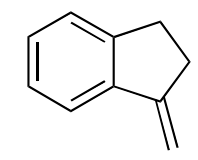

1-Methylene-2,3-dihydro-1H-indene (Table 2, entry 9) [1194-56-5].

Chromatography (100\% pentane) furnished the desired product as a colorless oil (contaminated with 5\% 1,2-divinylbenzene). Run 1: 45.5 mg (70\%); Run 2: $46.2 \mathrm{mg}$ (71\%).

${ }^{1} \mathrm{H}$ NMR $\left(\mathrm{CDCl}_{3}, 300 \mathrm{MHz}\right) \delta$ 7.54-7.45 (m, 1H), 7.31-7.19 (m, 3H), $5.45(\mathrm{t}, J=2.5 \mathrm{~Hz}$, $1 \mathrm{H}), 5.04(\mathrm{t}, J=2.2 \mathrm{~Hz}, 1 \mathrm{H}), 3.02-2.97(\mathrm{~m}, 2 \mathrm{H}), 2.84-2.76(\mathrm{~m}, 2 \mathrm{H})$;

${ }^{13} \mathrm{C}$ NMR $\left(\mathrm{CDCl}_{3}, 75 \mathrm{MHz}\right) \delta 150.8,147.0,141.3,128.5,126.7,125.6,120.9,102.7,31.4$, 30.2;

IR (film) 3066, 2917, 2850, 1715, 1639, 1462, 1441, 1400, 1381, 1279, 1169, 1017, 1003, $945,914,868,771,718 \mathrm{~cm}^{-1}$;

GCMS (EI) calcd for $\mathrm{C}_{10} \mathrm{H}_{10} 130[\mathrm{M}]^{+}$, found 130 .

General procedure for alkyl chlorides (Table 3). An oven-dried 25-mL Schlenk flask was purged with argon for $10 \mathrm{~min}$, then charged with $\mathrm{Pd}_{2}(\mathrm{MeO}-\mathrm{dba})_{3}(27.4 \mathrm{mg}$, $0.025 \mathrm{mmol}, 0.050$ equiv), SIMes $\cdot \mathrm{HBF}_{4}(39.4 \mathrm{mg}, 0.10 \mathrm{mmol}, 0.20$ equiv), $\mathrm{KO} t$ - $\mathrm{Bu}$ (11.2 $\mathrm{mg}$, $0.10 \mathrm{mmol}, 0.20$ equiv), and $\mathrm{K}_{3} \mathrm{PO}_{4}(117 \mathrm{mg}, 0.55 \mathrm{mmol}, 1.1$ equiv). The flask was then purged with argon for an additional $5 \mathrm{~min}$, with stirring. Dry NMP $(5 \mathrm{~mL})$ was added, and the mixture was stirred under argon for $10 \mathrm{~min}$. The electrophile was then added via a gastight syringe $(0.50 \mathrm{mmol}, 1.0$ equiv), and the Schlenk flask was sealed. The reaction mixture was stirred for $16 \mathrm{~h}$ at $100{ }^{\circ} \mathrm{C}$. Next, the mixture was allowed to cool to room temperature, and then $\mathrm{H}_{2} \mathrm{O}(100 \mathrm{~mL})$ and $\mathrm{Et}_{2} \mathrm{O}(50 \mathrm{~mL})$ were added. The aqueous layer was extracted with $\mathrm{Et}_{2} \mathrm{O}(3 \times 30 \mathrm{~mL})$, and the combined organic layers were washed with $\mathrm{H}_{2} \mathrm{O}(100 \mathrm{~mL})$ and brine $(30 \mathrm{~mL})$, dried $\left(\mathrm{MgSO}_{4}\right)$, filtered, and concentrated. The residue was purified by chromatography $\left(10 \% \mathrm{w} / \mathrm{w} \mathrm{AgNO}_{3}\right.$ on $\mathrm{SiO}_{2}$, $10 \mathrm{~g} / \mathrm{mmol}$ product).

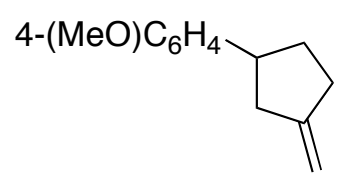

\section{1-Methoxy-4-(3-methylenecyclopentyl)benzene (Table 3, entry 1).}

Chromatography (50:1 hexanes: $\left.\mathrm{Et}_{2} \mathrm{O}\right)$ furnished the desired product as a colorless oil. Run 1: $69.6 \mathrm{mg}$ (74\%); Run 2: $65.8 \mathrm{mg}(70 \%)$.

For characterization data, see Table 2, entry 3. 


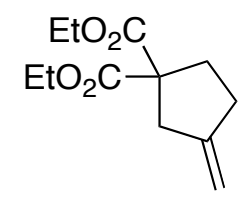

Diethyl 3-methylenecyclopentane-1,1-dicarboxylate (Table 3, entry 2) [137435-462]. Chromatography (20:1 hexanes: $\left.\mathrm{Et}_{2} \mathrm{O}\right)$ furnished the desired product as a colorless oil. Run 1: 92.7 mg (82\%); Run 2: 88.1 mg (78\%).

For characterization data, see Table 2, entry 6.

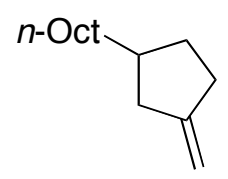

1-Methylene-3-octylcyclopentane (Table 3, entry 3). Chromatography (100\% hexanes) furnished the desired product as a colorless oil. Run 1: $67.9 \mathrm{mg}(70 \%)$; Run 2: $69.8 \mathrm{mg}(72 \%)$.

For characterization data, see Table 2, entry 7.

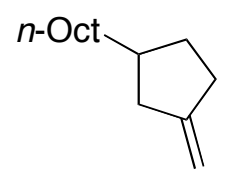

1-Methylene-3-octylcyclopentane (Table 3, entry 4). Chromatography (100\% hexanes) furnished the desired product as a colorless oil. Run 1: $65.0 \mathrm{mg}$ (67\%); Run 2: $62.1 \mathrm{mg}(64 \%)$.

For characterization data, see Table 2, entry 7.

\section{Eq 2}<smiles>[2H]C=Cc1ccccc1Br</smiles>

(E)-1-Bromo-2-(2-deuterovinyl)benzene. Prepared by the procedure of Soderquist ${ }^{3}$ by hydroboration of 2'-bromophenylacetylene $(2.94 \mathrm{~g}, 16.0 \mathrm{mmol}, 1.0$ equiv) with 9-BBN dimmer (1.95 g, $8.0 \mathrm{mmol}, 0.50$ equiv), followed by protonolysis with AcOD. Product: $1.85 \mathrm{~g}(62 \%)$.

${ }^{1} \mathrm{H} \mathrm{NMR}\left(\mathrm{CDCl}_{3}, 300 \mathrm{MHz}\right)$ 8 7.59-7.54 (m, 2H), 7.33-7.26 (m, 1H), 7.16-7.10 (m, 1H), $7.07(\mathrm{~d}, J=17.5 \mathrm{~Hz}, 1 \mathrm{H}), 5.71(\mathrm{~d}, J=17.5 \mathrm{~Hz}, 1 \mathrm{H}) ;{ }^{2} \mathrm{H}$ incorporation: $94 \%$. 


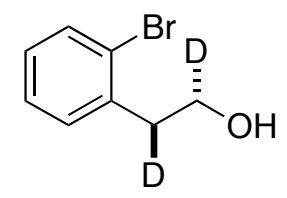

2-(2-Bromophenyl)-1,2-dideuteroethanol. $\mathrm{NaBD}_{4}(462 \mathrm{mg}, 11 \mathrm{mmol})$ was added to an oven-dried 100-mL round-bottom flask, which was then purged with argon for 5 min. A solution of the styrene derivative $(1.8 \mathrm{~g}, 10 \mathrm{mmol})$ in THF $(20 \mathrm{~mL})$ was added, followed by $\mathrm{BF}_{3} \mathrm{OEt}_{2}$ (over $10 \mathrm{~min}$ ). The reaction mixture was heated to $40{ }^{\circ} \mathrm{C}$ and stirred for $3 \mathrm{~h}$. Next, the mixture was cooled to $0{ }^{\circ} \mathrm{C}$, and $\mathrm{H}_{2} \mathrm{O}(4.5 \mathrm{~mL})$ was carefully added, followed by an aqueous solution of $\mathrm{NaOH}(3 \mathrm{~N} ; 5 \mathrm{~mL})$ and $\mathrm{H}_{2} \mathrm{O}_{2}(30 \% ; 5 \mathrm{~mL})$. The reaction mixture heated to $50{ }^{\circ} \mathrm{C}$ and stirred for $2 \mathrm{~h}$. Then, it was allowed to cool to room temperature, and $\mathrm{NaCl}(4 \mathrm{~g})$ was added, followed by $\mathrm{Et}_{2} \mathrm{O}(100 \mathrm{~mL})$ and brine (50 $\mathrm{mL})$. The organic layer was separated and then washed with $\mathrm{H}_{2} \mathrm{O}(50 \mathrm{~mL})$, dried $\left(\mathrm{MgSO}_{4}\right)$, filtered, and concentrated. The residue was purified by column chromatography (4:1 hexanes: $\left.\mathrm{Et}_{2} \mathrm{O}\right)$, which furnished $800 \mathrm{mg}(40 \%$; not optimized) of the desired product.

${ }^{1} \mathrm{H}$ NMR $\left(\mathrm{CDCl}_{3}, 500 \mathrm{MHz}\right) \delta 7.57(\mathrm{~d}, J=7.8 \mathrm{~Hz}, 1 \mathrm{H}), 7.30-7.24(\mathrm{~m}, 2 \mathrm{H}), 7.13-7.09(\mathrm{~m}$, $1 \mathrm{H}), 3.88(\mathrm{t}, J=6.0 \mathrm{~Hz}, 1 \mathrm{H}), 3.02(\mathrm{~d}, J=6.5 \mathrm{~Hz}, 1 \mathrm{H}), 1.42(\mathrm{~s}, 1 \mathrm{H})$;

${ }^{13} \mathrm{C} \mathrm{NMR}\left(\mathrm{CDCl}_{3}, 75 \mathrm{MHz}\right) \delta 138.4,133.7,132.0,128.9,128.2,125.4,62.4(\mathrm{t}, J=23.0$ $\mathrm{Hz}), 39.6(\mathrm{t}, J=19.5 \mathrm{~Hz})$;

IR (film) 3356, 3057, 2918, 2165, 1566, 1470, 1437, 1263, 1098, 1022, $746 \mathrm{~cm}^{-1}$; GCMS (EI) calcd for $\mathrm{C}_{8} \mathrm{H}_{7} \mathrm{D}_{2} \mathrm{BrO} 202 / 204[\mathrm{M}]^{+}$, found 202/ 204 (1:1).

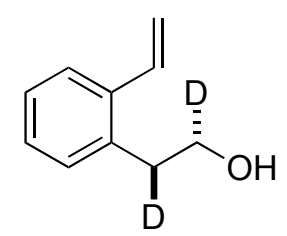

2-(2-Vinylphenyl)-1,2-dideuteroethanol. The title compound was prepared according to a general procedure for Stille cross-couplings. ${ }^{4}$ The alcohol from the previous step ( $800 \mathrm{mg}, 3.94 \mathrm{mmol}, 1.00$ equiv), tributylvinyltin $(1.31 \mathrm{~g}, 4.13 \mathrm{mmol}, 1.05$ equiv), $\mathrm{Pd}_{2} \mathrm{dba}_{3}$ ( $36 \mathrm{mg}, 0.039 \mathrm{mmol}, 0.010$ equiv), and $\mathrm{P}(t-\mathrm{Bu})_{3}(17.5 \mathrm{mg}, 0.084 \mathrm{mmol}$, 0.020 equiv) were stirred in toluene $(4 \mathrm{~mL})$ under argon for $24 \mathrm{~h}$. Then, KF ( $3 \mathrm{~g})$ was added, followed by $\mathrm{Et}_{2} \mathrm{O}(10 \mathrm{~mL})$ and activated carbon $(3 \mathrm{~g})$. The mixture was stirred for $5 \mathrm{~min}$, and then it was filtered through a pad of silica (washed with $\mathrm{Et}_{2} \mathrm{O}(3 \times 20$ $\mathrm{mL})$ ). The filtrate was concentrated, and the residue was purified by column chromatography (5:1 hexanes:EtOAc), which furnished $520 \mathrm{mg}$ of the desired product $(88 \%)$. 
${ }^{1} \mathrm{H}$ NMR $\left(\mathrm{CDCl}_{3}, 300 \mathrm{MHz}\right)$ \& 7.57-7.49 (m, 1H), 7.31-7.18 (m, 3H), $7.02(\mathrm{dd}, J=17.4$ $\mathrm{Hz}, 11.0 \mathrm{~Hz}, 1 \mathrm{H}), 5.66(\mathrm{dd}, J=17.3 \mathrm{~Hz}, 1.4 \mathrm{~Hz}, 1 \mathrm{H}), 5.32(\mathrm{dd}, J=11.0 \mathrm{~Hz}, 1.4 \mathrm{~Hz}, 1 \mathrm{H})$, $3.79(\mathrm{t}, J=6.6 \mathrm{~Hz}, 1 \mathrm{H}), 2.95(\mathrm{~d}, J=7.0 \mathrm{~Hz}, 1 \mathrm{H}), 1.44(\mathrm{~d}, J=6.0 \mathrm{~Hz}, 1 \mathrm{H})$;

${ }^{13} \mathrm{C}$ NMR $\left(\mathrm{CDCl}_{3}, 75 \mathrm{MHz}\right) \delta 137.3,135.9,134.6,130.6,128.1,127.2,126.3,116.3,63.0$ $(\mathrm{t}, J=21.9 \mathrm{~Hz}), 36.2(\mathrm{t}, J=19.5 \mathrm{~Hz})$;

IR (film) 3346, 3087, 3063, 3027, 2922, 2166, 1834, 1626, 1484, 1450, 1416, 1332, 1098, 1024, 991, 914, $772 \mathrm{~cm}^{-1}$;

GCMS (EI) calcd for $\mathrm{C}_{10} \mathrm{H}_{10} \mathrm{D}_{2} \mathrm{O} 150[\mathrm{M}]^{+}$, found 150 .<smiles>[2H]C(Br)C([2H])c1ccccc1C=C</smiles>

1-(2-Bromo-1,2-dideuteroethyl)-2-vinylbenzene. $\mathrm{PPh}_{3} \mathrm{Br}_{2}(1.72 \mathrm{~g}, 4.1 \mathrm{mmol}, 1.3$ equiv) and imidazole ( $319 \mathrm{mg}, 4.7 \mathrm{mmol}, 1.5$ equiv) were added to an oven-dried 100$\mathrm{mL}$ round-bottom flask, which was then purged with argon for 5 min. Dry $\mathrm{CH}_{2} \mathrm{Cl}_{2}(15$ $\mathrm{mL}$ ) was added, and the mixture was cooled to $0{ }^{\circ} \mathrm{C}$ in an ice bath. A solution of the alcohol ( $470 \mathrm{mg}$, $3.1 \mathrm{mmol}, 1.0$ equiv) in $\mathrm{CH}_{2} \mathrm{Cl}_{2}(10 \mathrm{~mL})$ was added dropwise over 10 min. Next, the ice bath was removed, and the reaction mixture was stirred for $3 \mathrm{~h}$. After the reaction was complete according to $\mathrm{TLC}, \mathrm{SiO}_{2}(1.0 \mathrm{~g})$ was added directly to the mixture, which was stirred for $5 \mathrm{~min}$. The stir bar was removed, and the mixture was concentrated to a dry solid (careful: fine powder), then placed atop a column of silica gel, which was eluted with $100 \%$ hexanes to give the product (527 $\mathrm{mg}, 79 \%)$ as a colorless oil. As a precaution, this material was stored at $0{ }^{\circ} \mathrm{C}$.

${ }^{1} \mathrm{H} \mathrm{NMR}\left(\mathrm{CDCl}_{3}, 300 \mathrm{MHz}\right)$ 8 7.54-7.48 (m, 1H), 7.30-7.20 (m, 2H), 7.21-7.15 (m, 1H), $6.96(\mathrm{dd}, J=17.3 \mathrm{~Hz}, 11.0 \mathrm{~Hz}, 1 \mathrm{H}), 5.69(\mathrm{dd}, J=17.2 \mathrm{~Hz}, 1.3 \mathrm{~Hz}, 1 \mathrm{H}), 5.37(\mathrm{dd}, J=11.0$ $\mathrm{Hz}, 1.3 \mathrm{~Hz}, 1 \mathrm{H}), 3.48(\mathrm{~d}, J=5.9 \mathrm{~Hz}, 1 \mathrm{H}), 3.23(\mathrm{~d}, J=5.9 \mathrm{~Hz}, 1 \mathrm{H})$;

${ }^{13} \mathrm{C} \mathrm{NMR}\left(\mathrm{CDCl}_{3}, 75 \mathrm{MHz}\right) \delta 137.0,136.3,134.1,130.2,128.2,127.7,126.4,116.9,36.8$ $(\mathrm{t}, J=19.8 \mathrm{~Hz}), 31.9(\mathrm{t}, J=23.5 \mathrm{~Hz})$;

IR (film) 3087, 3063, 3027, 2211, 1922, 1839, 1626, 1484, 1450, 1417, 1327, 1191, 989, 916, 772, 730, $630 \mathrm{~cm}^{-1}$;

GCMS (EI) calcd for $\mathrm{C}_{10} \mathrm{H}_{9} \mathrm{D}_{2} \mathrm{Br} 212 / 214[\mathrm{M}]^{+}$, found 212/214 (1:1). 


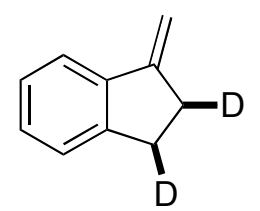

Eq 2. See the procedure for entry 9 of Table 2 (above). Run 1: $42.2 \mathrm{mg}(64 \%)$; Run 2: $43.6 \mathrm{mg}(66 \%)$.

${ }^{1} \mathrm{H}$ NMR $\left(\mathrm{CDCl}_{3}, 300 \mathrm{MHz}\right) \delta$ 7.54-7.48 (m, 1H), 7.31-7.18 (m, 3H), $5.46(\mathrm{~d}, J=2.5 \mathrm{~Hz}$, $1 \mathrm{H}), 5.04(\mathrm{~d}, J=2.2 \mathrm{~Hz}, 1 \mathrm{H}), 2.97(\mathrm{~d}, J=9.3 \mathrm{~Hz}, 1 \mathrm{H}), 2.78(\mathrm{dt}, J=9.4 \mathrm{~Hz}, 2.3 \mathrm{~Hz}, 1 \mathrm{H})$;

${ }^{2} \mathrm{H} \mathrm{NMR}\left(\mathrm{CDCl}_{3}, 77 \mathrm{MHz}\right) \delta 3.01(\mathrm{~d}, J=2.2 \mathrm{~Hz}, 1 \mathrm{D}), 2.83(\mathrm{~d}, J=2.6 \mathrm{~Hz}, 1 \mathrm{D})$;

${ }^{13} \mathrm{C} \mathrm{NMR}\left(\mathrm{CDCl}_{3}, 75 \mathrm{MHz}\right) \delta 150.9,147.0,141.4,128.5,126.7,125.6,120.8,102.7,31.0$ $\left(\mathrm{t}, J_{\mathrm{CD}}=20.2 \mathrm{~Hz}\right), 29.9\left(\mathrm{t}, J_{\mathrm{CD}}=19.9 \mathrm{~Hz}\right)$;

IR (film) 3070, 3020, 2917, 2851, 2169, 1715, 1638, 1462, 1275, 1019, 860, $758 \mathrm{~cm}^{-1}$; GCMS (EI) calcd for $\mathrm{C}_{10} \mathrm{H}_{8} \mathrm{D}_{2} 132[\mathrm{M}]^{+}$, found 132 . 


\section{References}

(1) Fairlamb, I. J. S.; Kapdi, A. R.; Lee, A. F. Org. Lett. 2004, 6, 4435-4438.

(2) Djakovitch, L.; Wagner, M.; Hartung, C. G.; Beller, M.; Koehler, K. J. Mol. Catal. A: Chem. 2004, 219, 121-130.

(3) Matos, K.; Soderquist, J. A. J. Org. Chem. 1998, 63, 461-470.

(4) Littke, A. F.; Schwarz, L.; Fu, G. C. J. Am. Chem. Soc. 2002, 124, 6343-6348. 


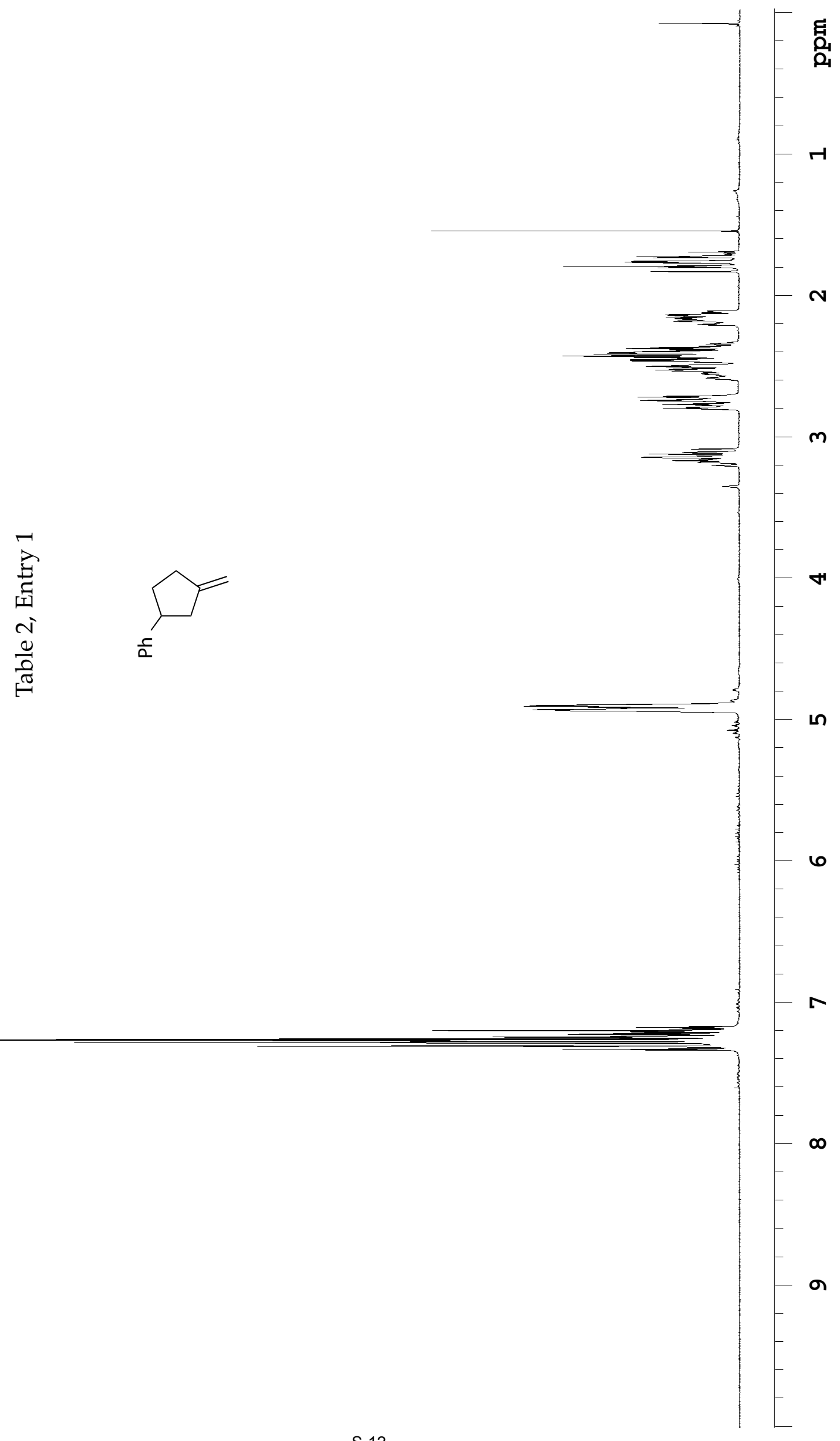




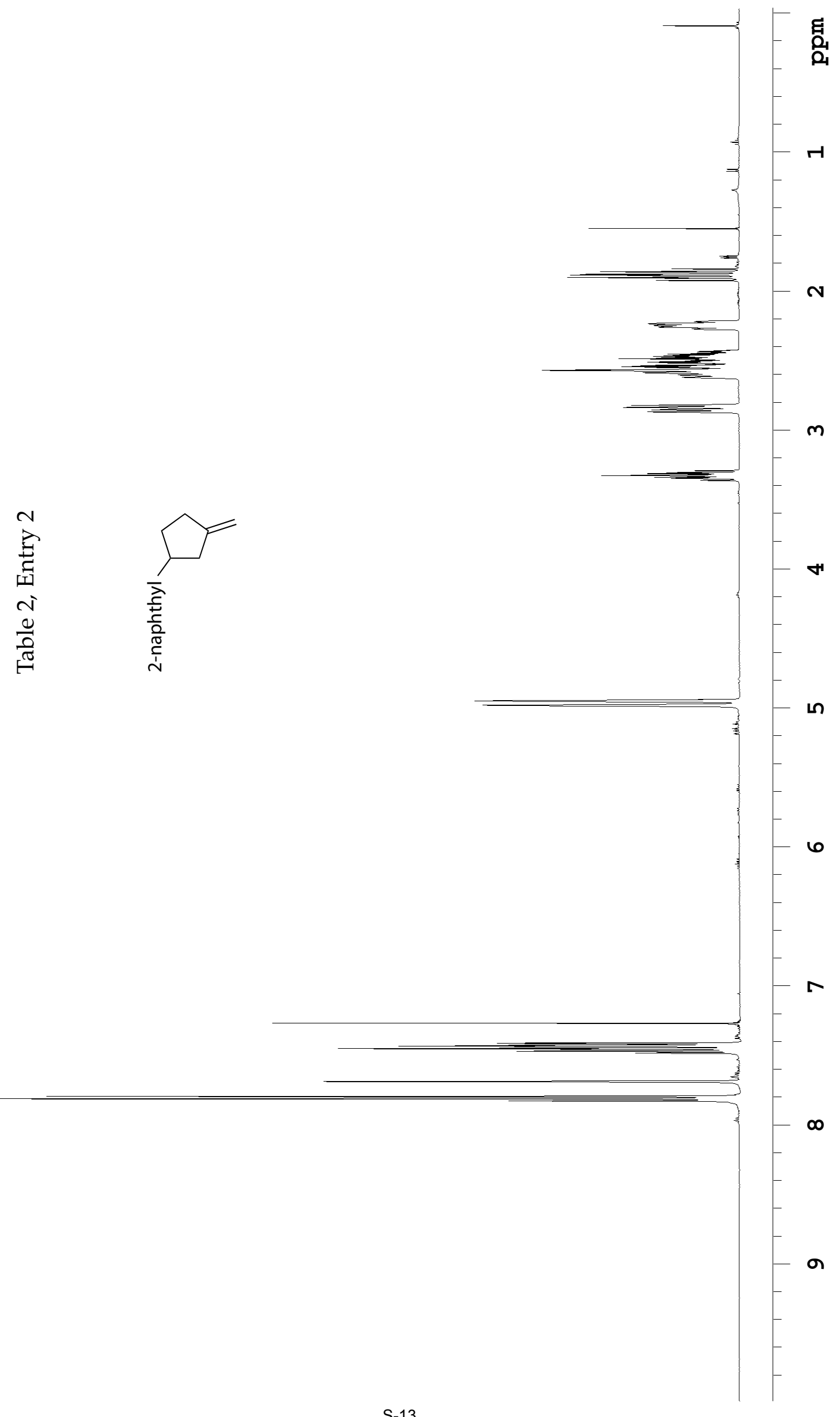




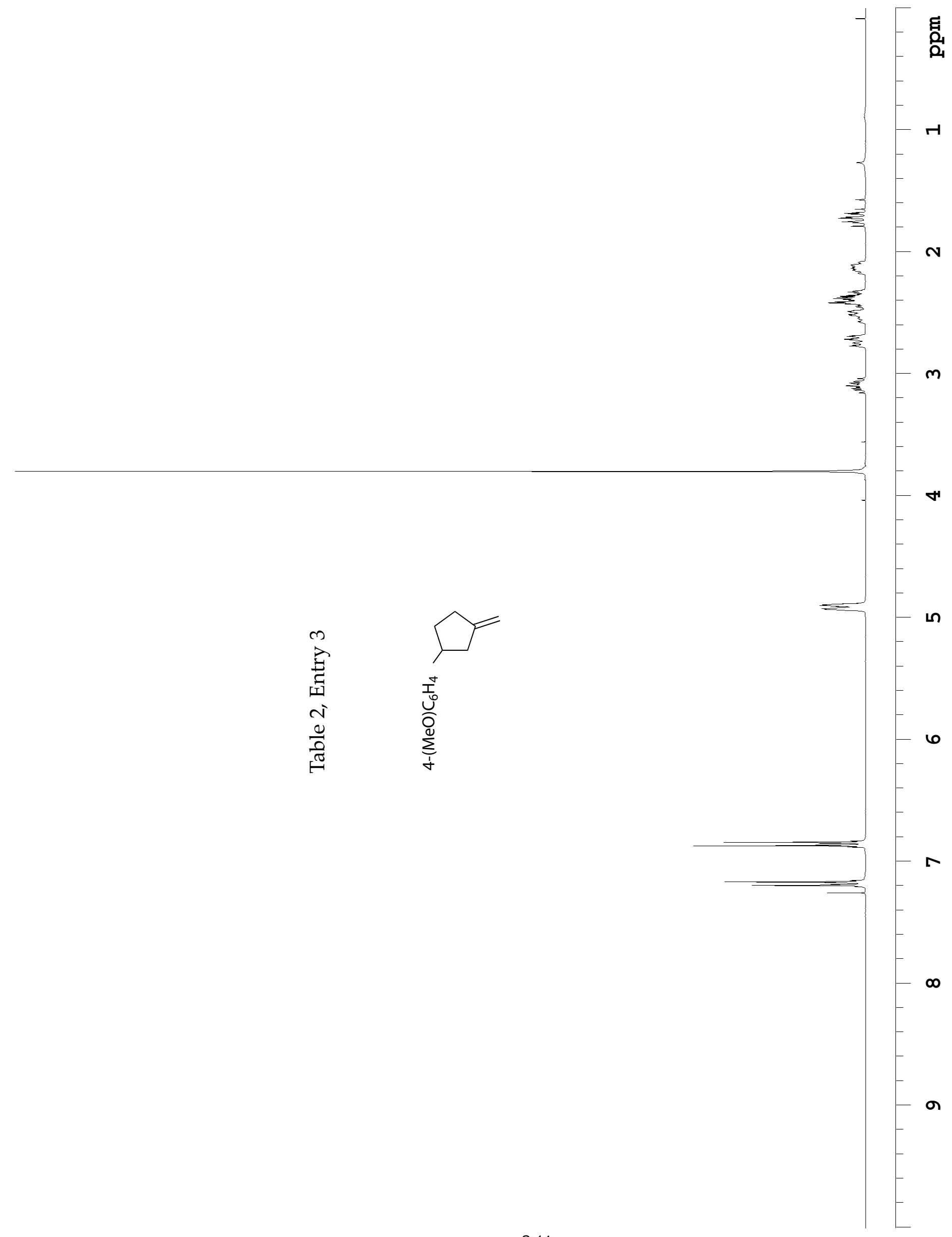




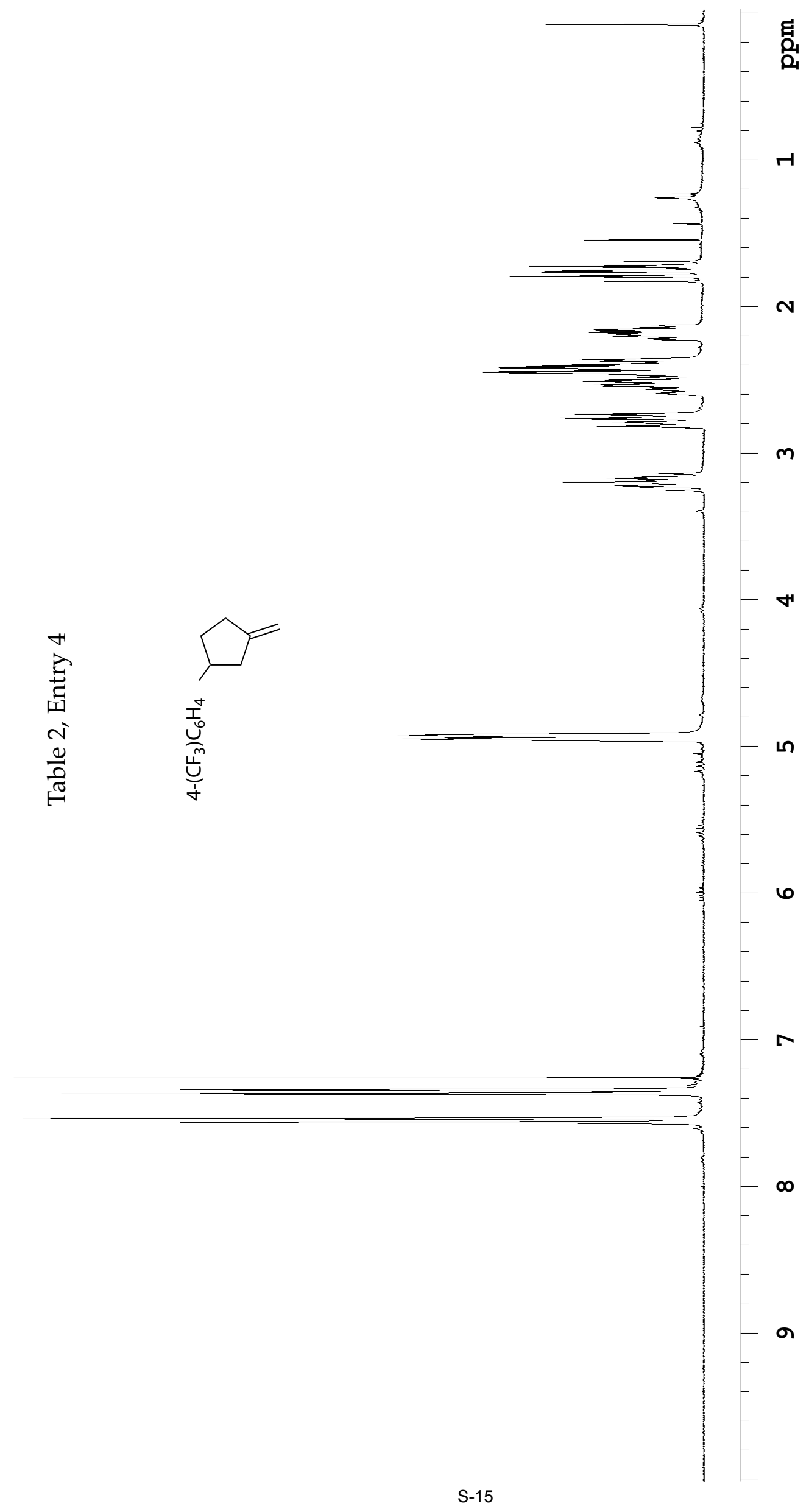




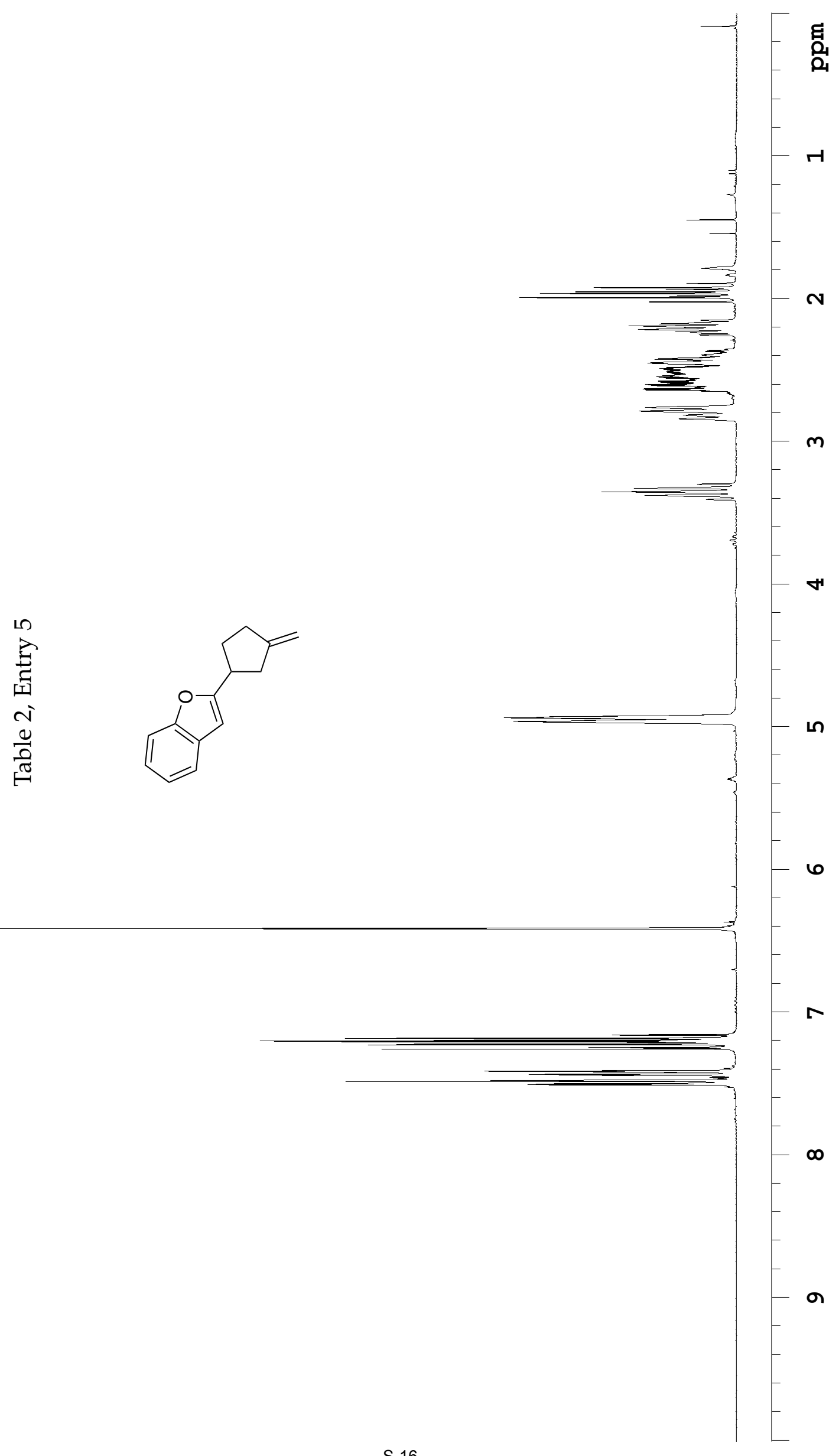




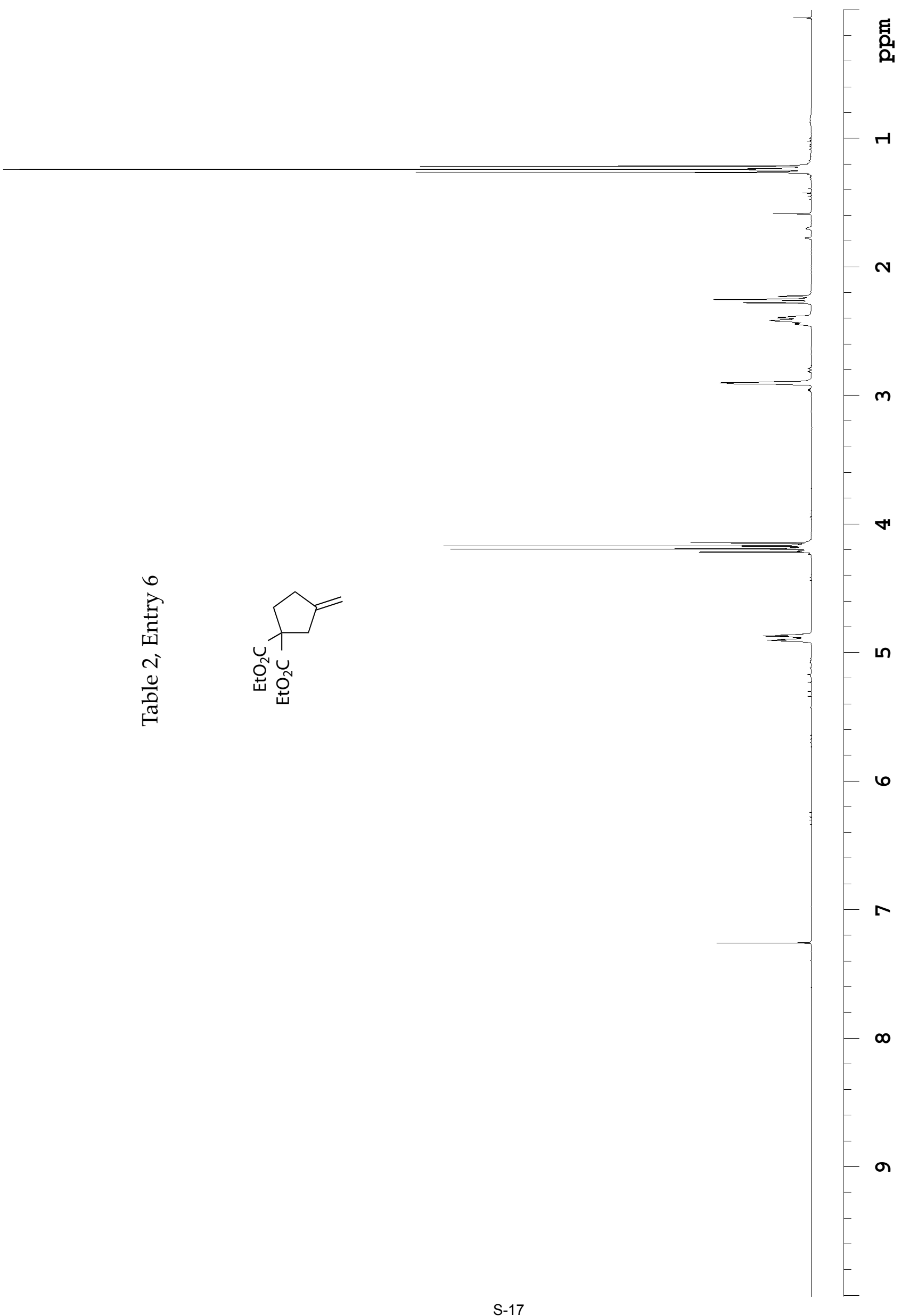




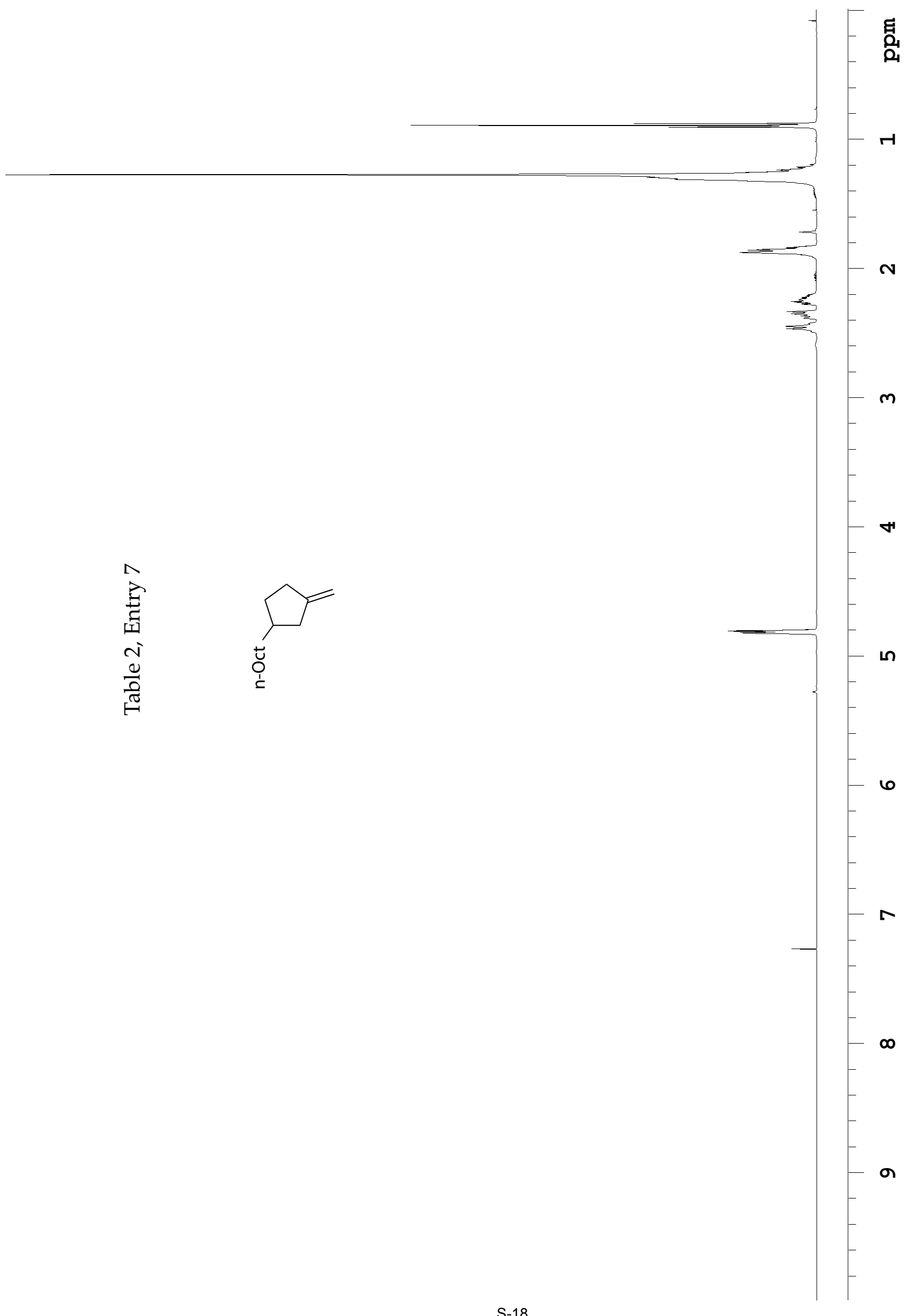




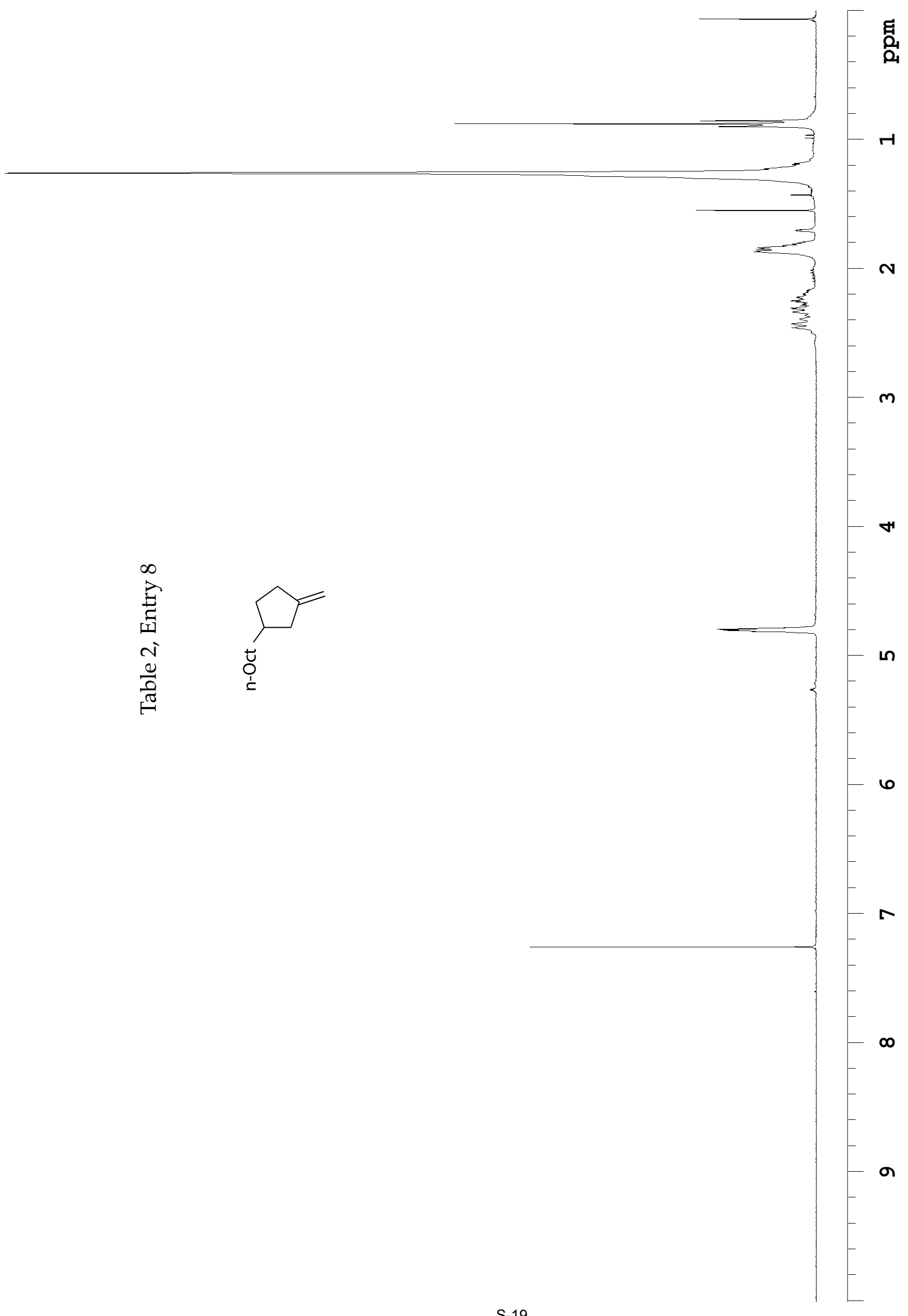


全

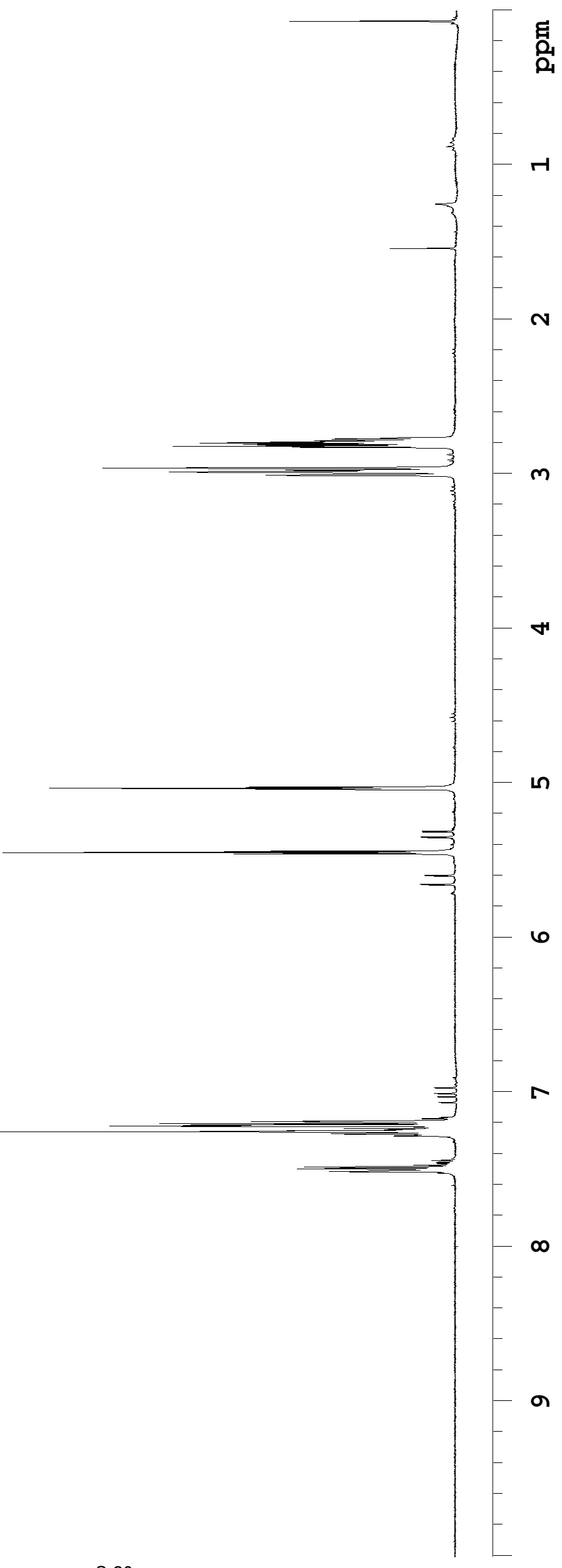




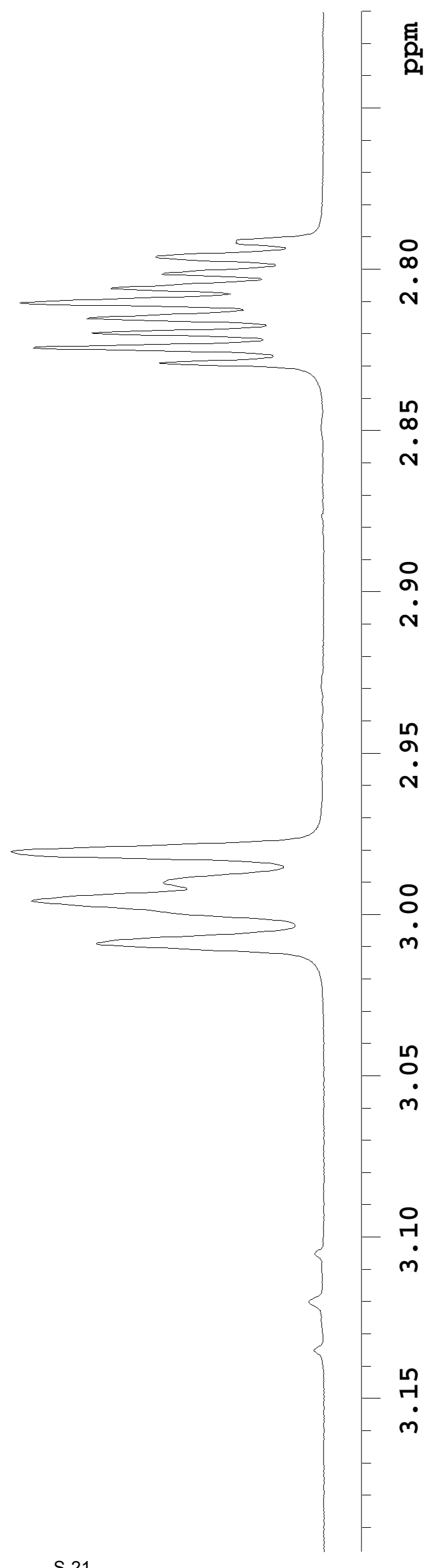




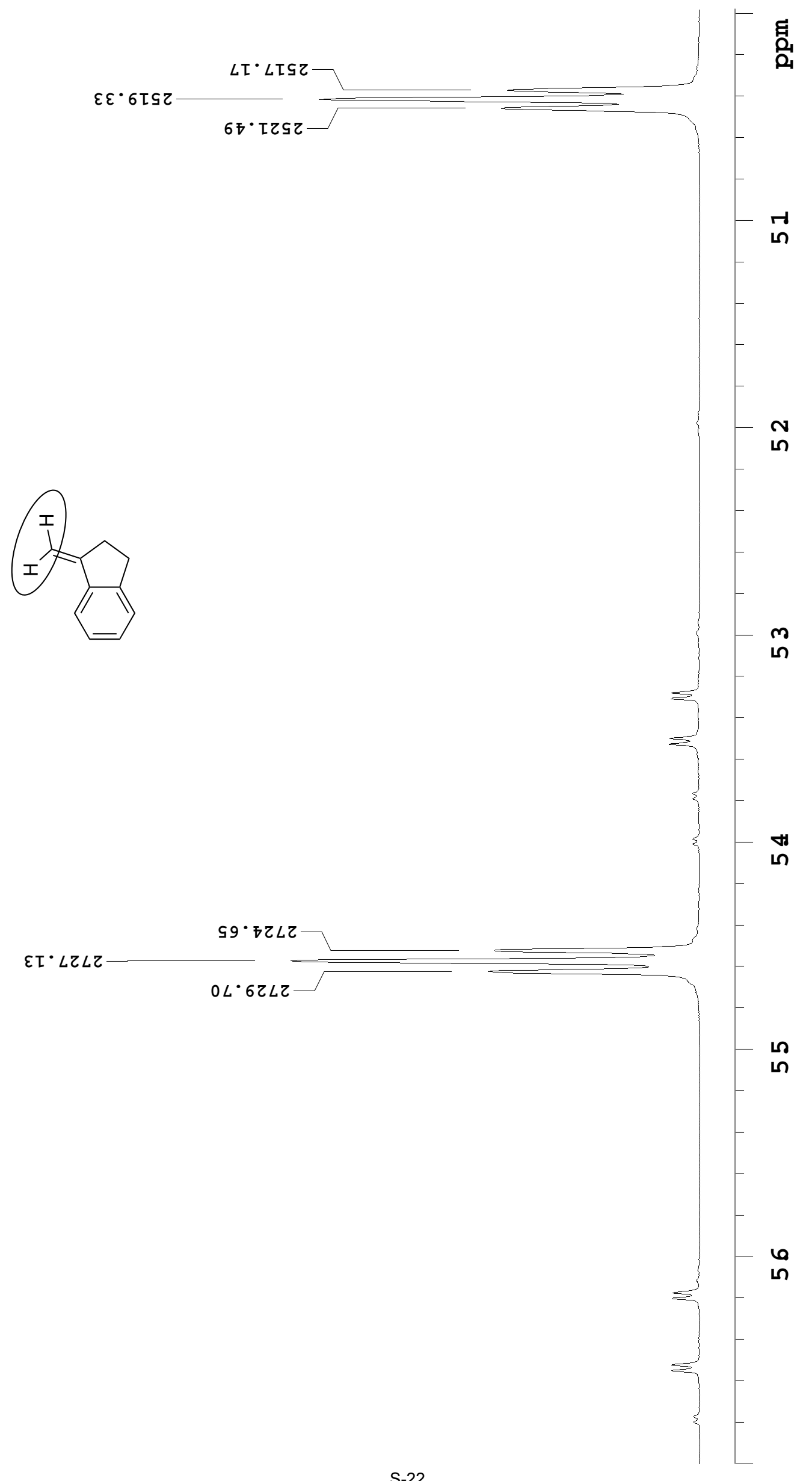




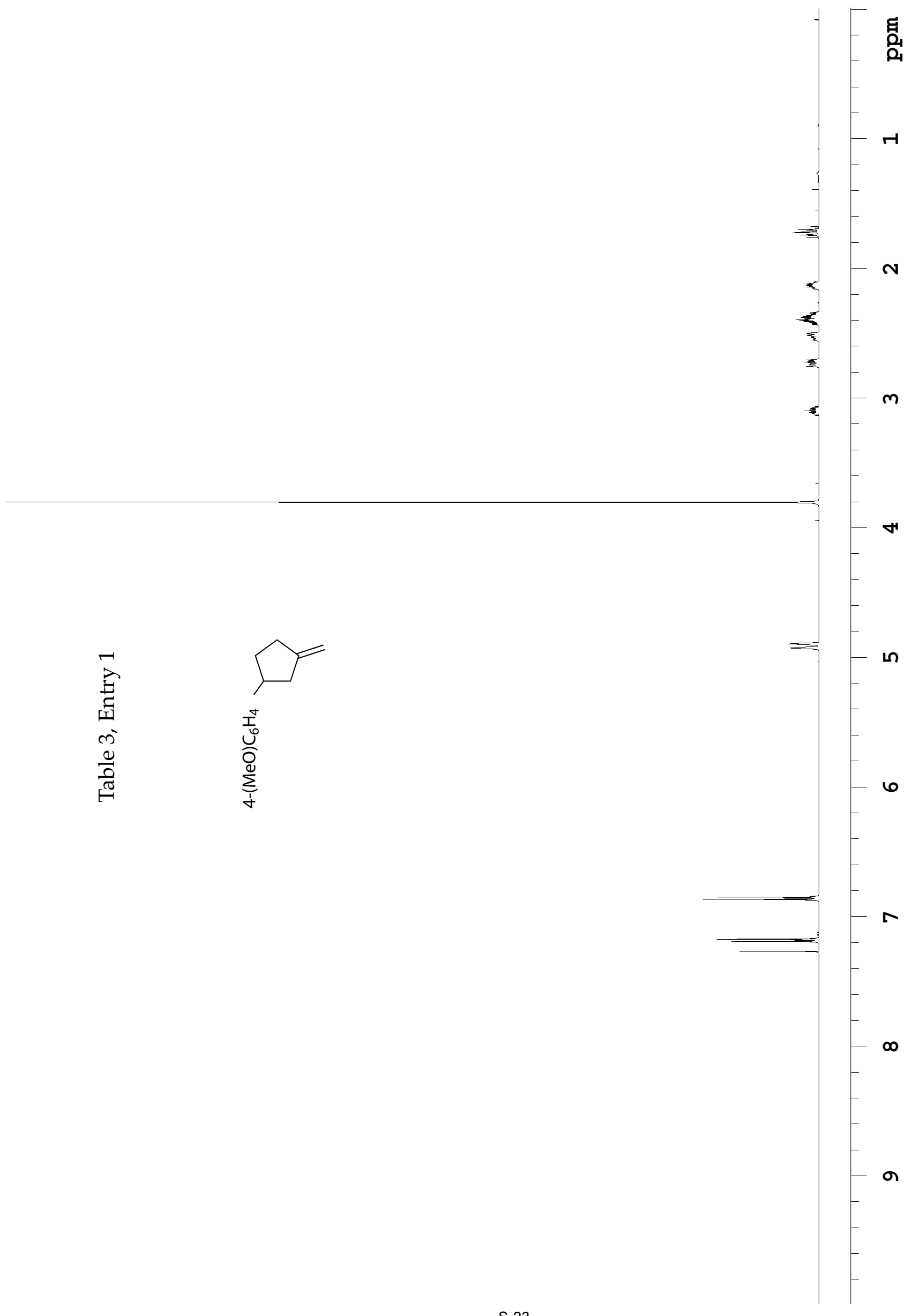




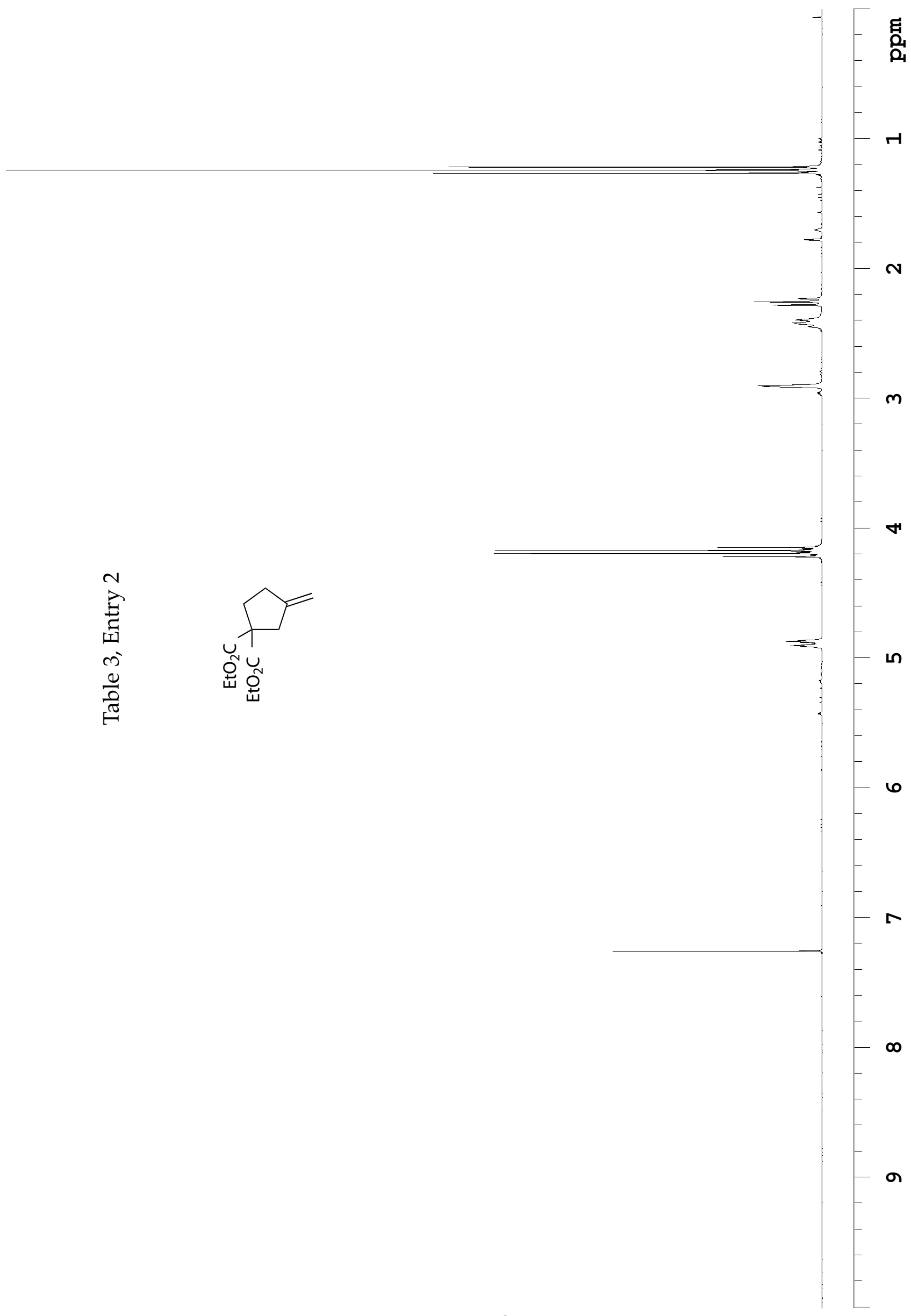

S-24 


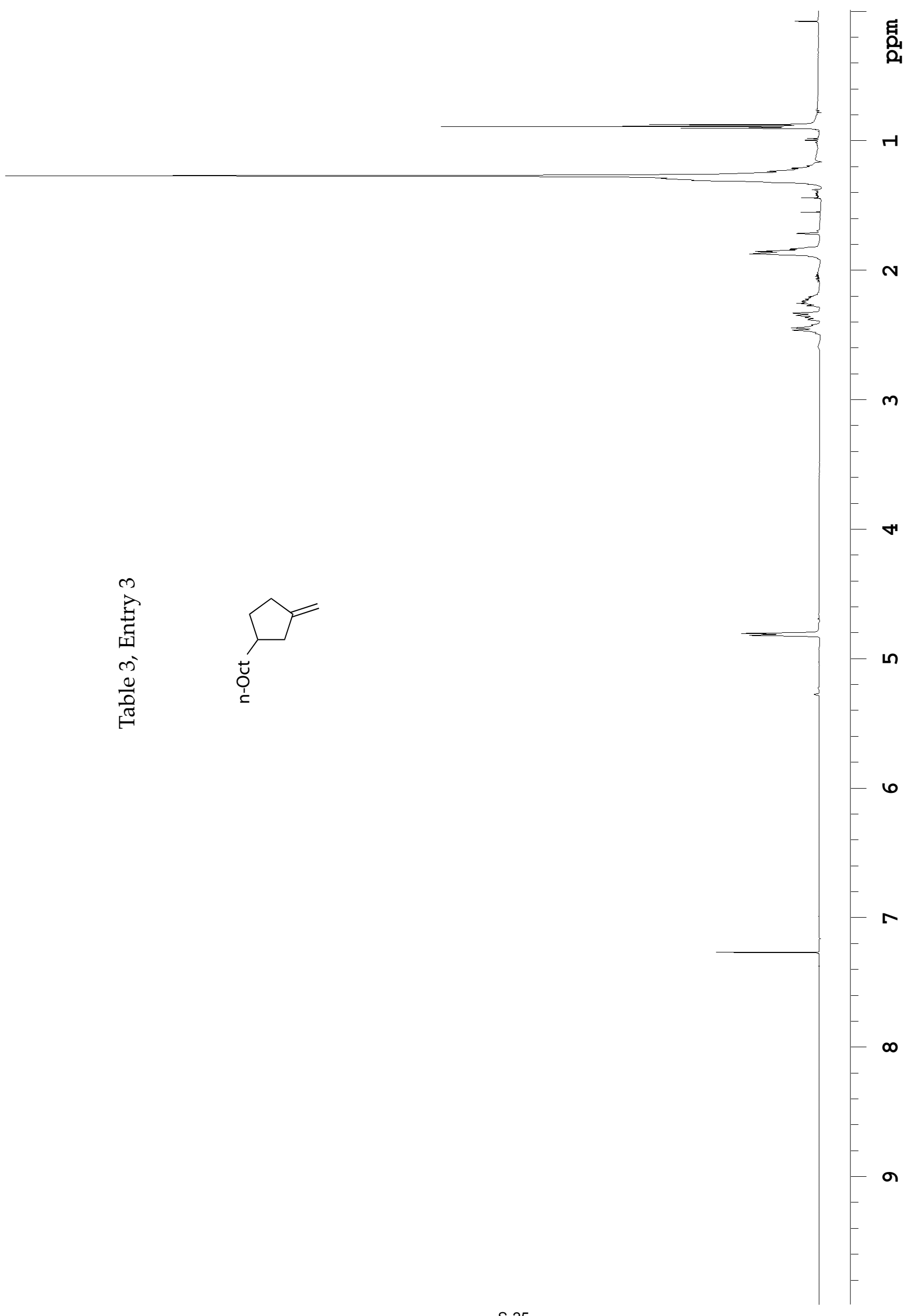




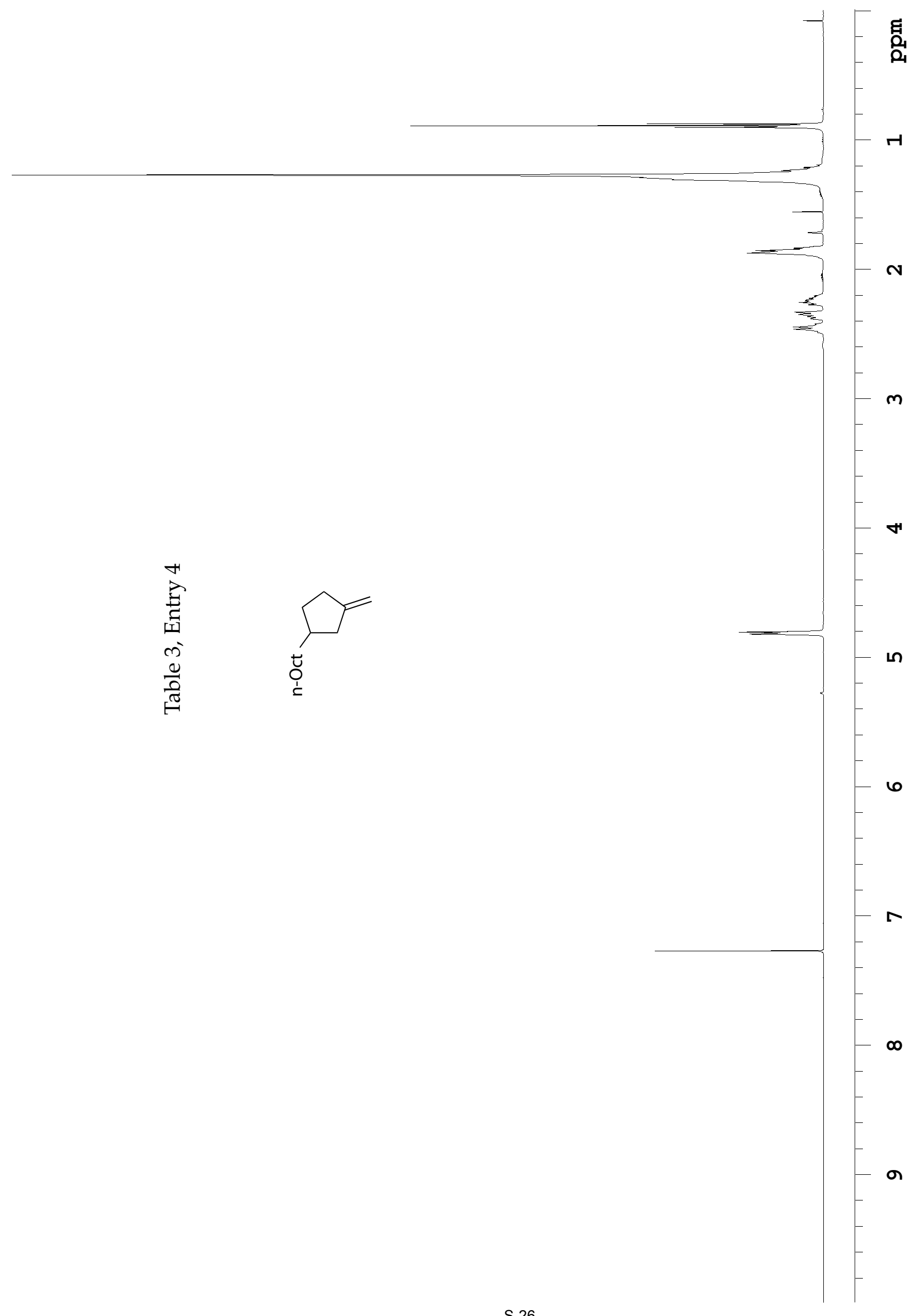




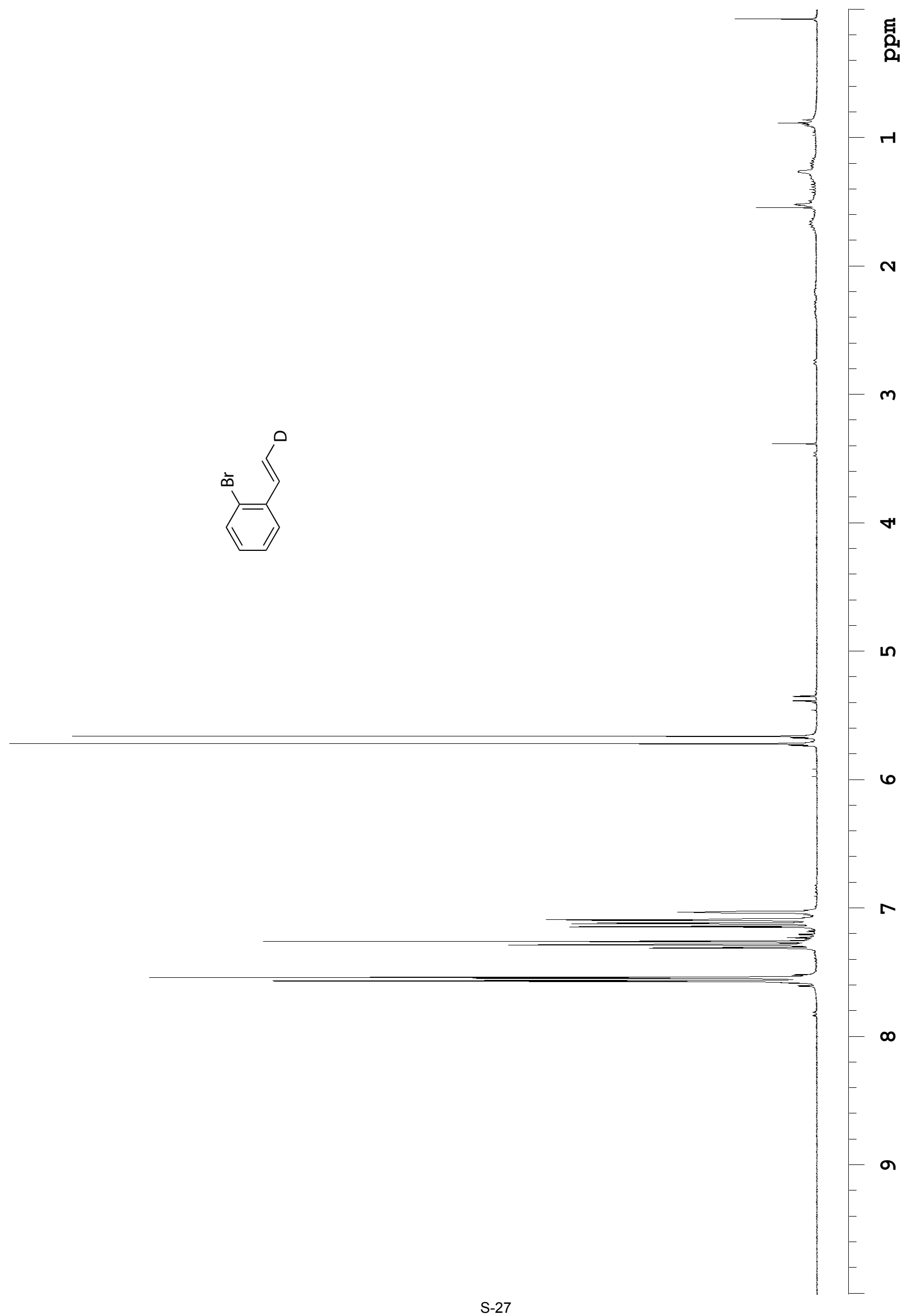




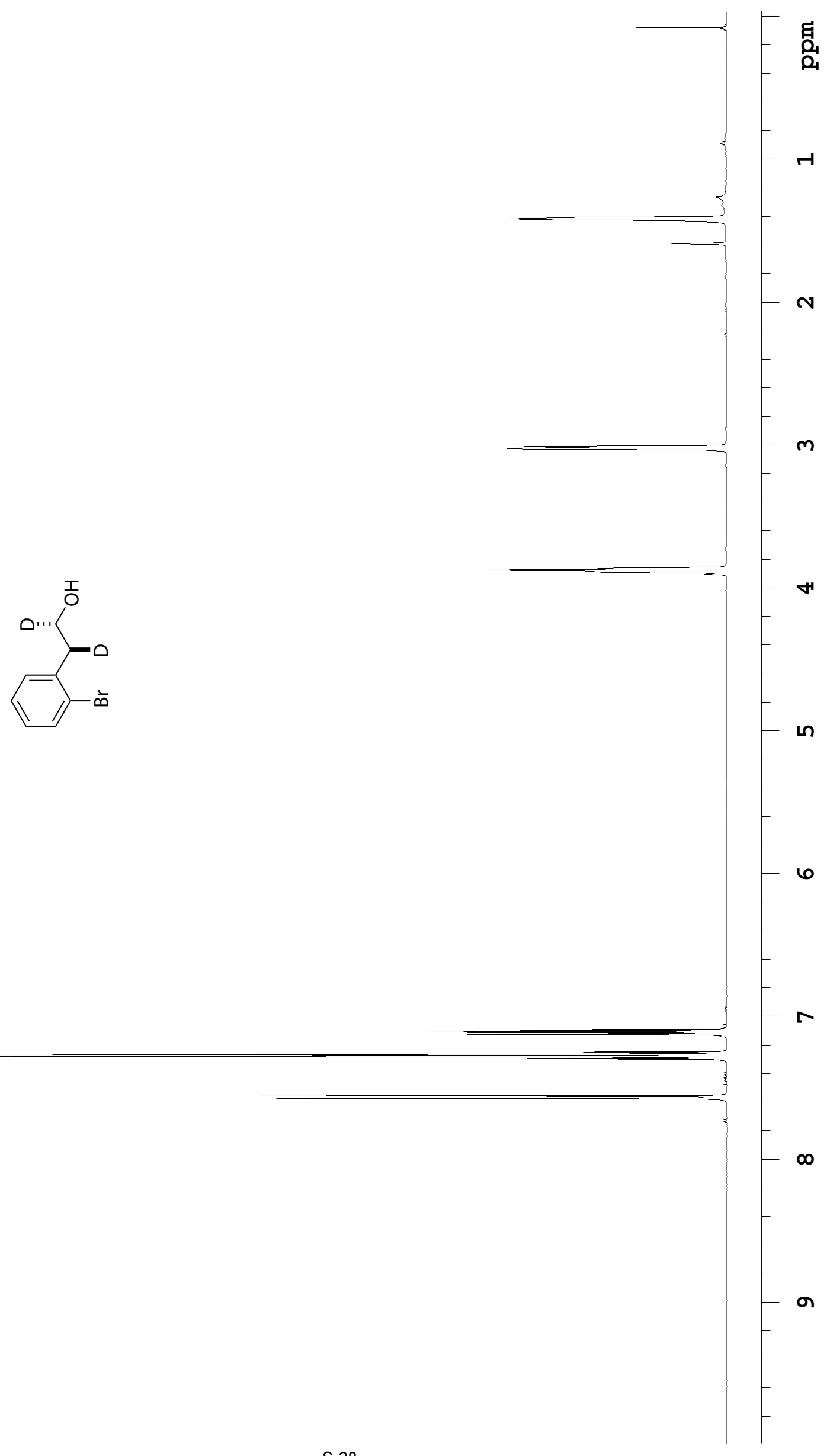




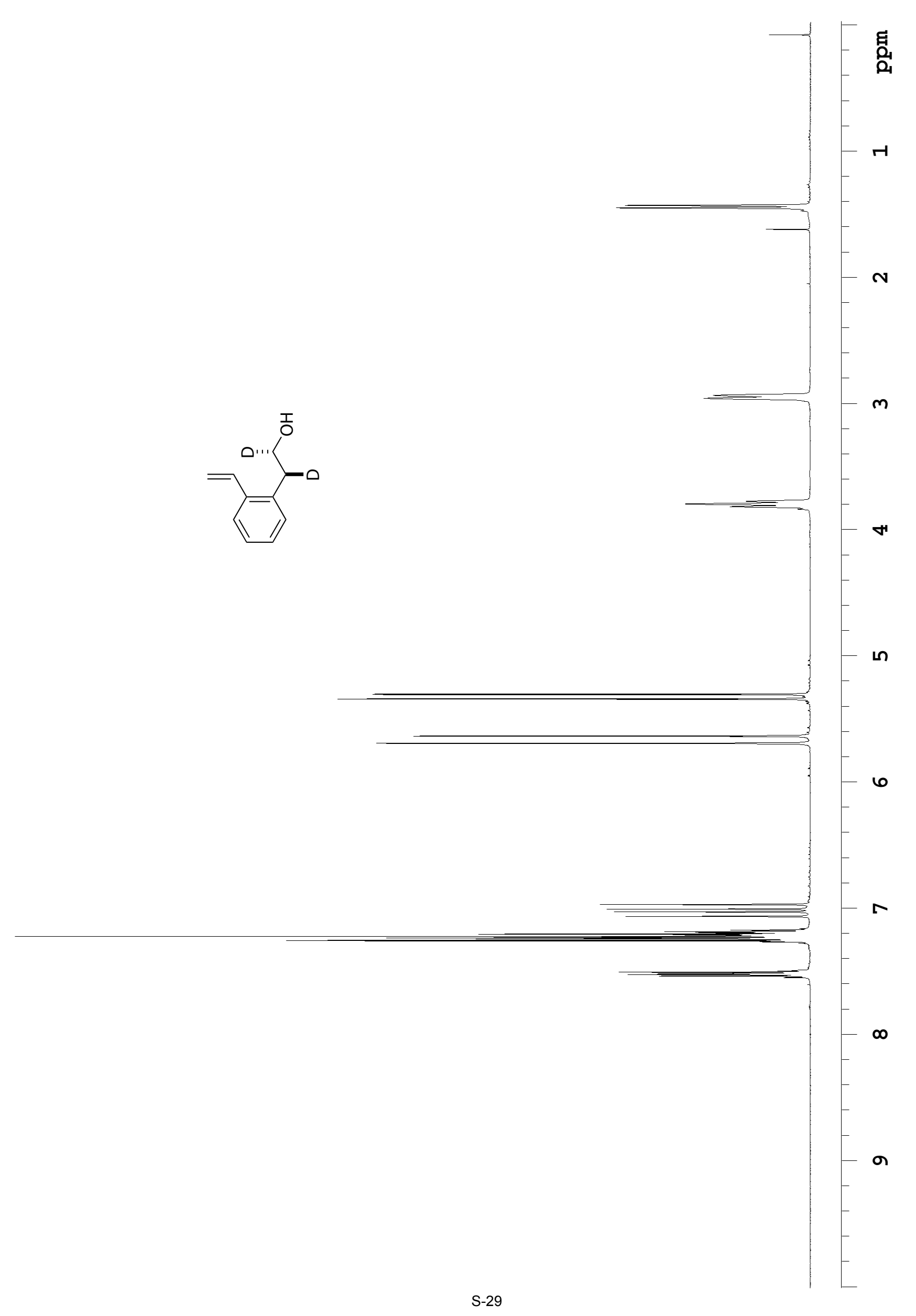




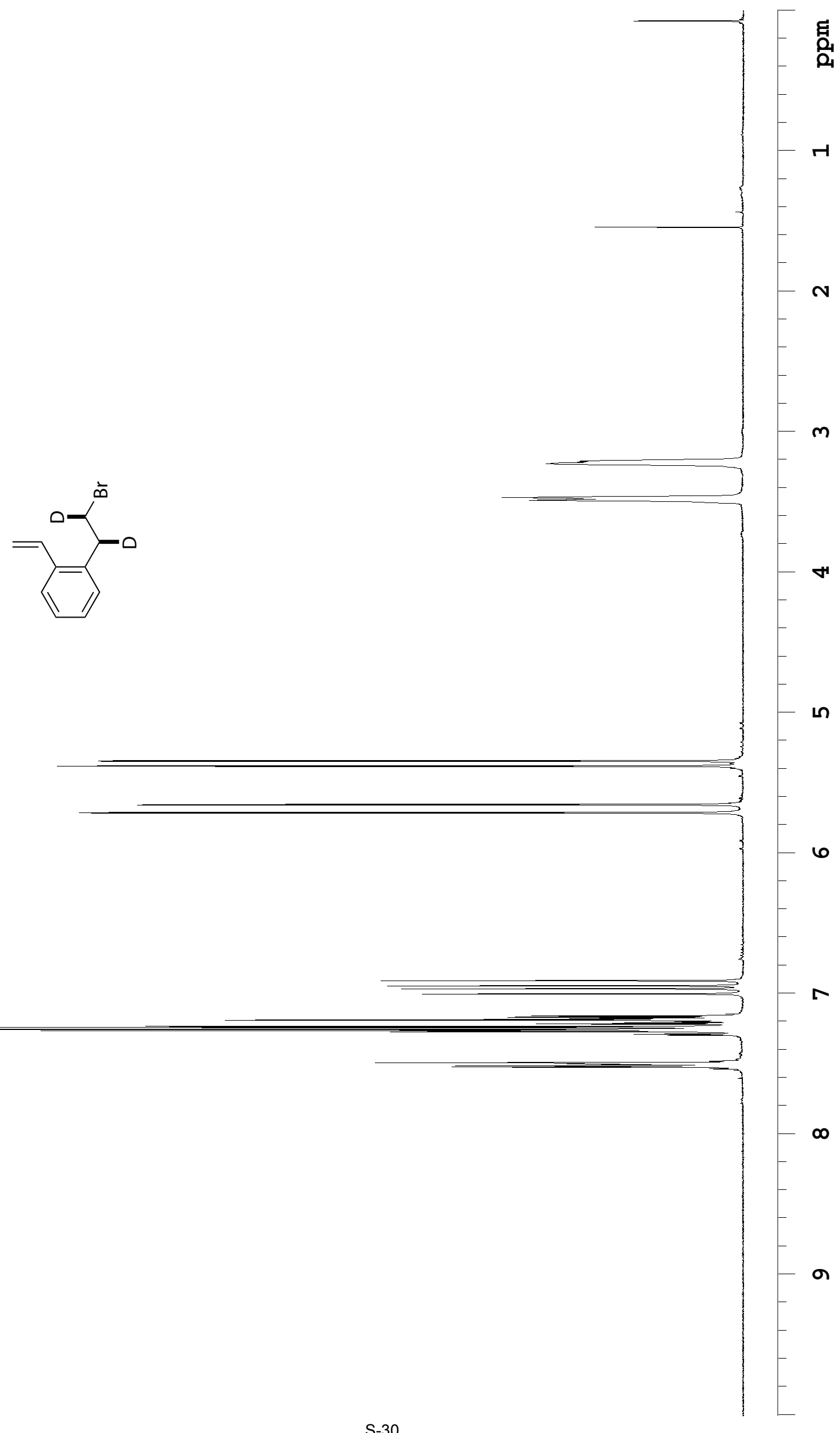




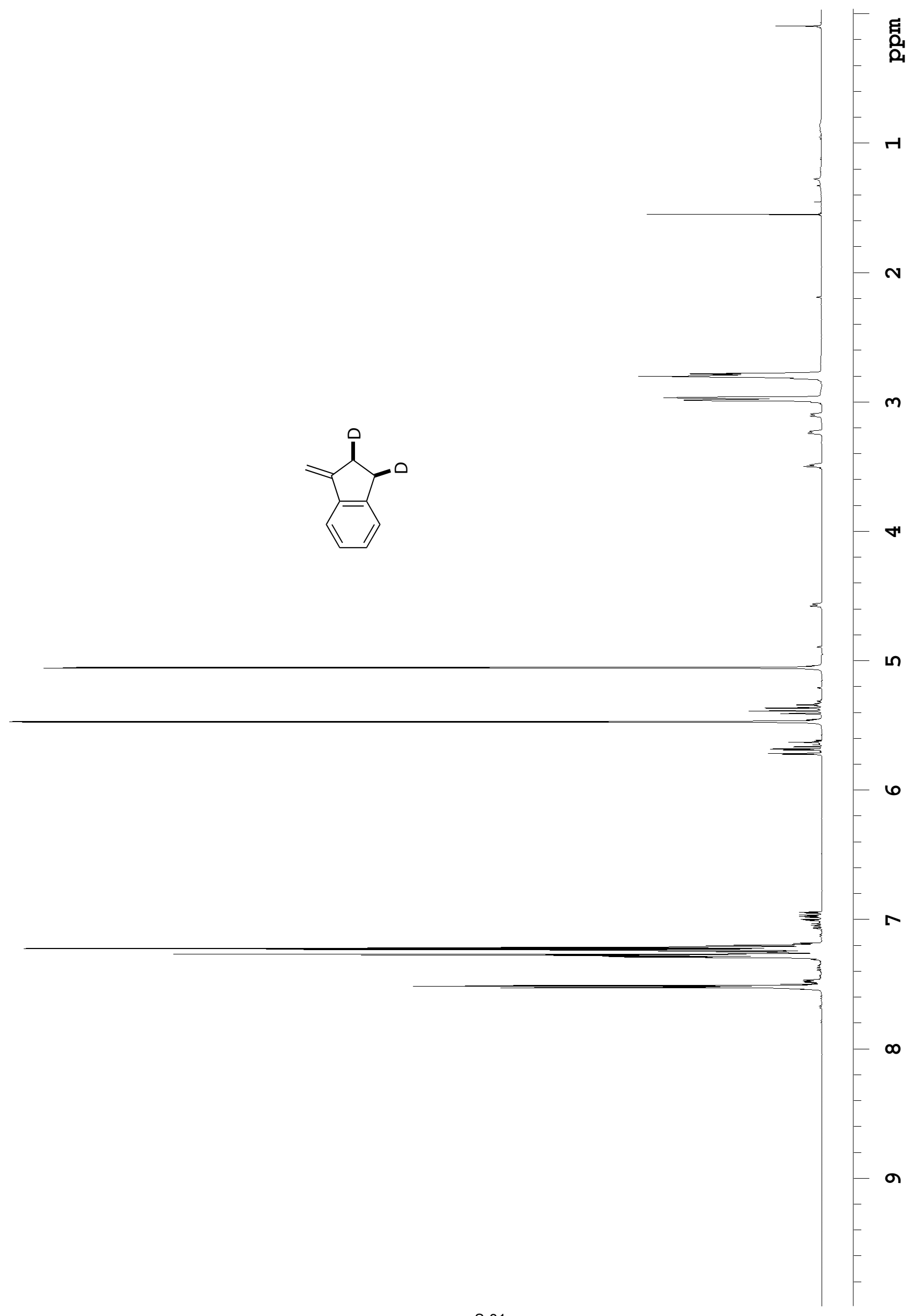




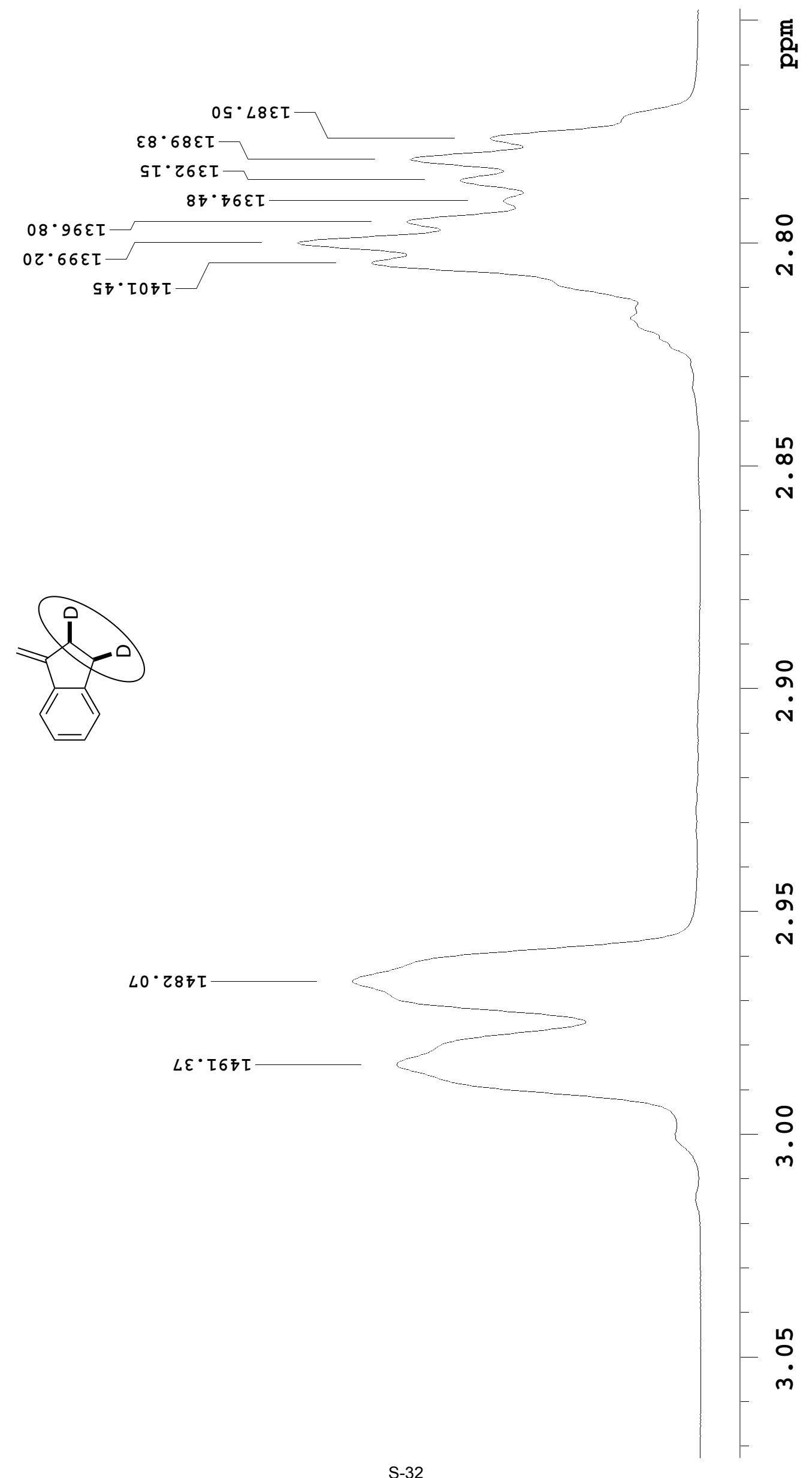



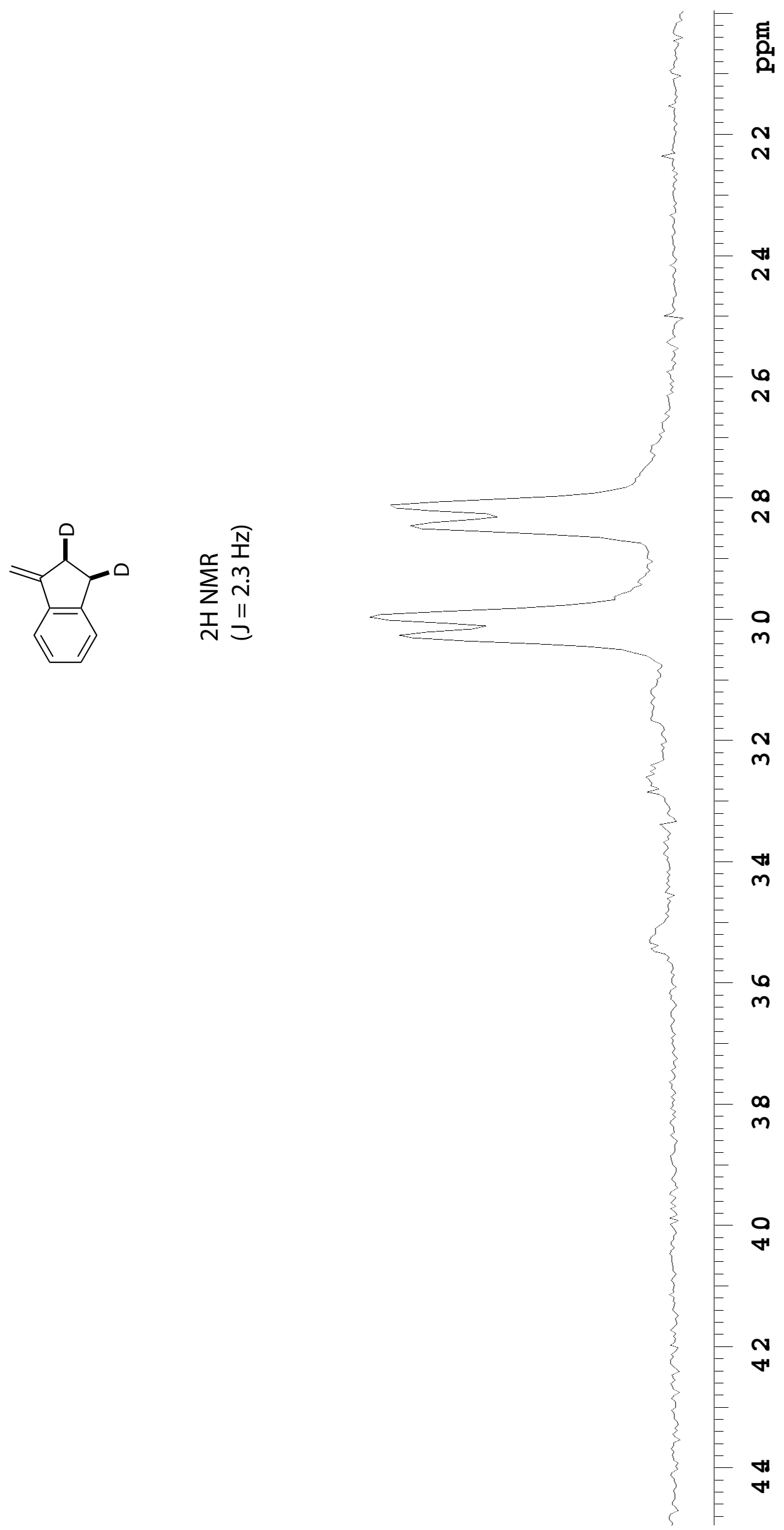


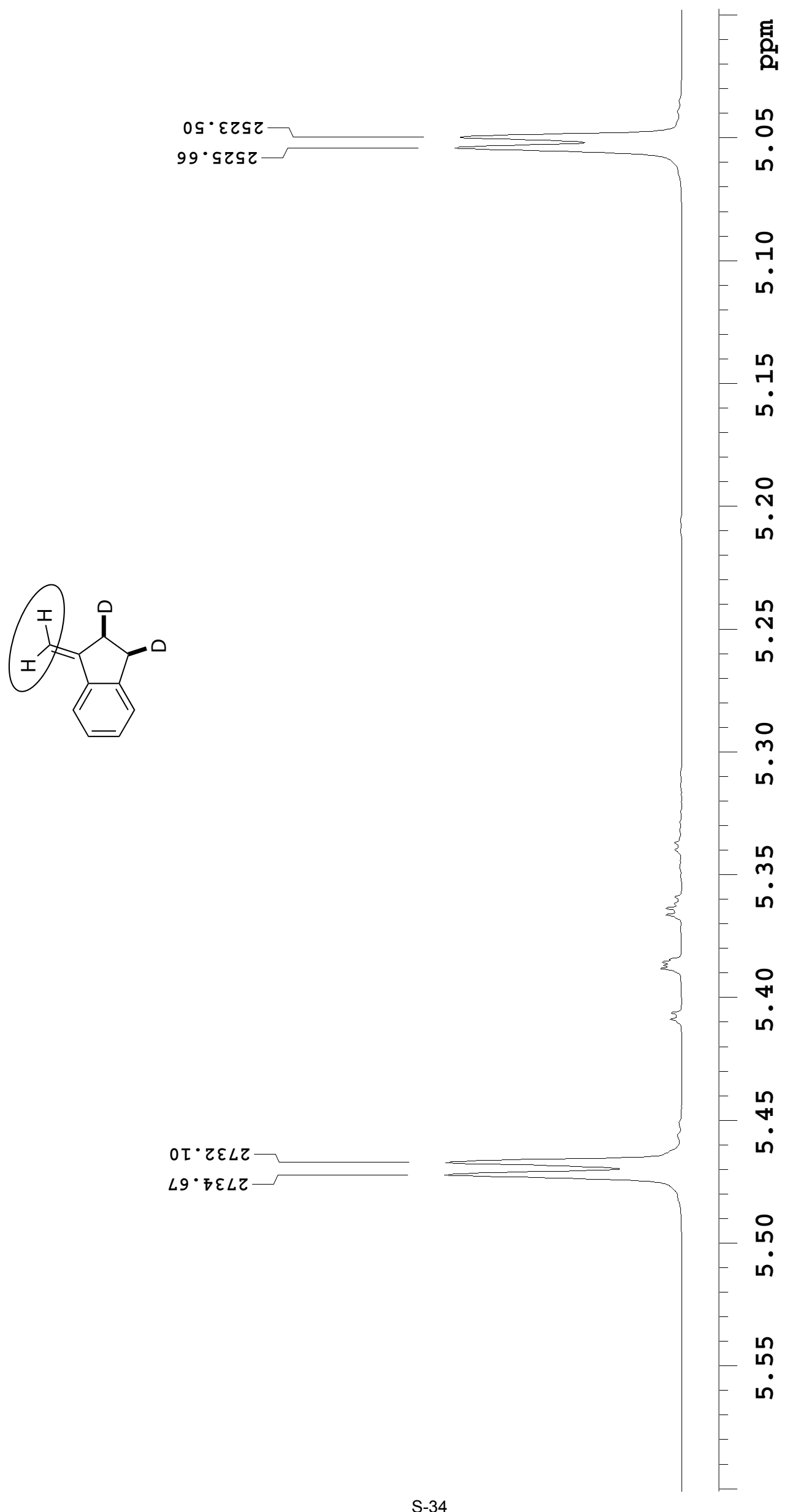

